\title{
Anisotropic chiral cosmology: exact solutions
}

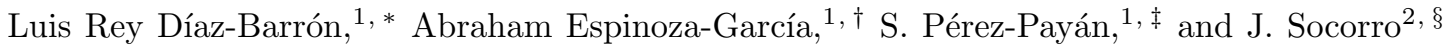 \\ ${ }^{1}$ Unidad Profesional Interdisciplinaria de Ingeniería, \\ Campus Guanajuato del Instituto Politécnico Nacional. \\ Av. Mineral de Valenciana \#200, Col. Fraccionamiento Industrial Puerto Interior, \\ C.P. 36275, Silao de la Victoria, Guanajuato, México. \\ ${ }^{2}$ Departamento de Física, DCeI, Universidad de Guanajuato-Campus León, C.P. 37150, León, Guanajuato, México
}

\begin{abstract}
In this work, we investigate the anisotropic Bianchi type I cosmological model in the chiral setup, in a twofold manner. Firstly, we consider a quintessence plus a k-essence like model, where two scalar fields but only one potential term is considered. Secondly, we look at a model where in addition to the two scalar fields the two potential terms are taken into account as well as the standard kinetic energy and the mixed term. Regarding this second model, it is shown that two possible cases can be studied: a quintom like case and a quintessence like case. In each of the models, we were able to find both classical and quantum analytical solutions.
\end{abstract}

PACS numbers: 98.80.Qc, 98.80.Es, 04.20.Jb

\section{INTRODUCTION}

It is well established that our universe is homogeneous and isotropic at large scales and can be modeled by the flat Friedman-Lemaittre-Robertson-Walker (FLRW) geometry. However, observations of the cosmic microwave background (CMB) have shown the existence of anomalies at large angular scales [1, this has led to put forward the hypothesis of primordial anisotropies in the early stages of the universe and could shed some light on the anomalies found in the CMB. Therefore, it is reasonable to address these issuess. Attempts to incorporate these ideas into the cosmological setting have been presented in Refs. 22 10, where anisotropic cosmological models have been used (mostly the Bianchi I model) as a background space-time in an early anisotropic but homogeneous universe that develops isotropization at the beginning of inflation, nonetheless, the imprints of such anisotropy would lead to the thermal maps of the CMB; and once the inflationary period ends, as a consequence of this isotropization, the universe acquires a FLRW geometry recovering the standard picture of the evolution of the universe. Due to the above, anisotropic cosmological models represent an attractive arena to test the early stages of the universe, even if no conclusive evidence that a primordial anisotropy is needed.

To tackle the different phenomena of our universe, scalar field cosmological models have been broadly used. Some of the issues that have been addressed under this line of thought are the dark matter component of the universe, the late time acceleration and the inflationary epoch, to name a few [11 25]. Although single scalar field cosmological models have been a cornerstone in giving answers to different problems present in our universe, there are still illnesses that haven't been cured. On this regard, in recent years cosmological models considering two or more scalar fields have drawn tremendous attention. The advantages of these models (compared to the single field ones) is the introduction of new degrees of freedom which allows the explanation of several physical phenomena.

Generally speaking, in this multi-scalar field cosmological models, the interaction of the scalar fields occur in the

\footnotetext{
*Electronic address: lrdiaz@ipn.mx

$\dagger$ Electronic address: aespinoza@ipn.mx

$\ddagger$ Electronic address: saperezp@ipn.mx

§Electronic address: socorro@fisica.ugto.mx
} 
potential, the mixed kinetic terms or both. In this setup, an inflationary picture of the universe can also be achieved [26, 27, even if the interaction between the scalar fields does not take place [28]. Moreover, multi-scalar fields models can also be used to explain the primordial inflation perturbations analysis [29, 30] or the assisted inflation [27, 31]. Another appealing reason to work with these models is that when two scalar fields are considered, the crossing of the cosmological constant boundary -1 can be described, in the litearature these models are known as quintom models [32 35] (single scalar field models do not have this malleability, since they only describe either the phantom or quintessence regime). Furthermore, this multi-field models can also tackle the hybrid inflation of the universe, which gives an alternative graceful exit in comparison to the standard inflationary picture [36 43]. The most successful models, phenomenologically speaking, are those that have incorporated quintessence scalar fields, slow-roll inflation, chiral cosmology connected with $f(R)$ theories and the nonlinear sigma model [18, 28, 44, 65].

In connection with the latter, multi-field anisotropic cosmological models of inflation have been explored. In Ref. 66] the author delves into the study of the Bianchi type I cosmology considering two interacting scalar fields and a potential of the form $V(\phi, \chi) \sim \phi^{4}+\chi^{4}$, founding numerical solutions as well as the asymptotically isotropic Friedmann case. Other interesting works are presented in Refs. [40] and 67], where the potential with structure $V(\phi, \sigma) \sim e^{\phi+\sigma}$ has shown to be a good viable candidate to address the inflationary era in both flat isotropic and anisotropic space-times. More recently, in Ref. 68 the authors present the case of the anisotropic Bianchi type I cosmology in the multi-field setup with a potential of the form $V_{0} e^{-\left(\lambda_{1} \phi_{1}+\cdots+\lambda_{n} \phi_{n}\right)}$, founding inflationary exact solutions in a quintessence framework. Additional research regarding multi-filed anisotropic cosmological models can be found in Refs. [55, 69, 78 .

In the present work we present the anisotropic Bianchi type I cosmological model with two scalar fields in a twofold manner. Following closely the developments introduced in Refs. 79] and [80, first, we put forward a simple quintessence plus a k-essence model which arises from considering the interaction of the two scalar fields but only one potential term. And second, a chiral approach is studied, in this case, in addition to the previous two scalar fields we also consider the two potential terms as well as the standard kinetic energy and the mixed term. For each model, classical and quantum analytical solutions are found.

This paper is arranged as follows. In section II we introduce the first model, where the Einstein-Klein-Gordon (EKG) equations are calculated and the Lagrangian and Hamiltonian approach is implemented in order to find the corresponding solutions as well as the anisotropic parameters. In section III. the second model is presented, here, after obtaining the Hamiltonian density we can distinguish two possible scenarios: a quintom like case and a quintessence case. For both scenarios the corresponding solutions are found. Section IV is devoted to implement the quantum versions of the previous two models and the corresponding solutions are obtained. Finally, section $\mathrm{V}$ is left for the final remarks.

\section{FIRST MODEL: QUINTESSENCE PLUS K-ESSENCE}

As we already mentioned, we are going to start by analyzing the quintessence plus k-essence model. For this purpose let us consider the Lagrangian density for such a model, which reads

$$
\mathcal{L}=\sqrt{-\mathrm{g}}\left(\mathrm{R}-\frac{1}{2} \mathrm{~g}^{\mu \nu} \nabla_{\mu} \phi_{1} \nabla_{\nu} \phi_{1}-\frac{1}{2} \mathrm{~g}^{\mu \nu} \nabla_{\mu} \phi_{2} \nabla_{\nu} \phi_{2}+\mathrm{V}\left(\phi_{1}\right)\right)
$$

where $\mathrm{R}$ is the Ricci scalar, $\mathrm{V}\left(\phi_{1}\right)=\mathrm{V}_{1} \mathrm{e}^{-\lambda_{1} \phi_{1}}$ is the corresponding scalar field potential (as it will be shown below), and the reduced Planck mass $M_{P}^{2}=1 / 8 \pi G=1$. The corresponding variations of (1), with respect to the metric and 
the scalar fields give the EKG field equations

$$
\begin{aligned}
\mathrm{G}_{\alpha \beta}= & -\frac{1}{2}\left(\nabla_{\alpha} \phi_{1} \nabla_{\beta} \phi_{1}-\frac{1}{2} \mathrm{~g}_{\alpha \beta} \mathrm{g}^{\mu \nu} \nabla_{\mu} \phi_{1} \nabla_{\nu} \phi_{1}\right)+\frac{1}{2} \mathrm{~g}_{\alpha \beta} \mathrm{V}\left(\phi_{1}\right) \\
& -\frac{1}{2}\left(\nabla_{\alpha} \phi_{2} \nabla_{\beta} \phi_{2}-\frac{1}{2} \mathrm{~g}_{\alpha \beta} \mathrm{g}^{\mu \nu} \nabla_{\mu} \phi_{2} \nabla_{\nu} \phi_{2}\right), \\
\square \phi_{1}-\frac{\partial \mathrm{V}}{\partial \phi_{1}}= & \mathrm{g}^{\mu \nu} \phi_{1, \mu \nu}-\mathrm{g}^{\alpha \beta} \Gamma_{\alpha \beta}^{\nu} \nabla_{\nu} \phi_{1}-\frac{\partial \mathrm{V}}{\partial \phi_{1}}=0, \\
& \mathrm{~g}^{\mu \nu} \phi_{2, \mu \nu}-\mathrm{g}^{\alpha \beta} \Gamma_{\alpha \beta}^{\nu} \nabla_{\nu} \phi_{2}=0 .
\end{aligned}
$$

The line element for the anisotropic cosmological Bianchi type I model in the Misner parametrization is

$$
\begin{aligned}
\mathrm{ds}^{2} & =-\mathrm{N}^{2} \mathrm{dt}^{2}+\mathrm{a}_{1}^{2} \mathrm{dx}^{2}+\mathrm{a}_{2}^{2} \mathrm{dy}^{2}+\mathrm{a}_{3}^{2} \mathrm{dz}^{2}, \\
& =-\mathrm{N}^{2} \mathrm{dt}^{2}+\mathrm{e}^{2 \Omega}\left[\mathrm{e}^{2 \beta_{+}+2 \sqrt{3} \beta_{-}} \mathrm{dx}^{2}+\mathrm{e}^{2 \beta_{+}-2 \sqrt{3} \beta_{-}} \mathrm{dy}^{2}+\mathrm{e}^{-4 \beta_{+}} \mathrm{dz}^{2}\right],
\end{aligned}
$$

where $\mathrm{a}_{\mathrm{i}}(\mathrm{i}=1,2,3)$ are the scale factors in directions $(\mathrm{x}, \mathrm{y}, \mathrm{z})$, respectively, and $\mathrm{N}$ is the lapse function. For convenience, and in order to carry out the analytical calculations, we consider the following representation for the line element (5)

$$
\mathrm{ds}^{2}=-\mathrm{N}^{2} \mathrm{dt}^{2}+\eta^{2}\left[\mathrm{~m}_{1}^{2} \mathrm{dx}^{2}+\mathrm{m}_{2}^{2} \mathrm{dy}^{2}+\mathrm{m}_{3}^{2} \mathrm{dz}^{2}\right]
$$

where the relations between both representations $(5)$ and $(6)$ are given by

$$
\begin{aligned}
\eta & =\mathrm{e}^{\Omega}, & & \dot{m}_{1} \\
\mathrm{~m}_{1} & =\mathrm{e}^{\beta_{+}+\sqrt{3} \beta_{-}}, & & \frac{\dot{\beta}_{+}}{\mathrm{m}_{1}} \sqrt{3} \dot{\beta}_{-}, \\
\mathrm{m}_{2} & =\mathrm{e}^{\beta_{+}-\sqrt{3} \beta_{-}}, & & \frac{\dot{\mathrm{m}}_{2}}{\mathrm{~m}_{2}}=\dot{\beta}_{+}-\sqrt{3} \dot{\beta}_{-}, \\
\mathrm{m}_{3} & =\mathrm{e}^{-2 \beta_{+}}, & & \frac{\dot{\mathrm{m}}_{3}}{\mathrm{~m}_{3}}=-2 \dot{\beta}_{+},
\end{aligned}
$$

and $\eta$ is a function that has information regarding the isotropic scenario and the $\mathrm{m}_{\mathrm{i}}$ are dimensionless functions that have information about the anisotropic behavior of the universe, such that

$$
\prod_{\mathrm{i}=1}^{3} \mathrm{~m}_{\mathrm{i}}=1, \quad \prod_{\mathrm{i}=1}^{3} \mathrm{a}_{\mathrm{i}}=\eta^{3}, \quad \sum_{\mathrm{i}=1}^{3} \frac{\dot{\mathrm{m}}_{\mathrm{i}}}{\mathrm{m}_{\mathrm{i}}}=0,
$$

act as constraint equations for the model.

\section{A. General Solutions to the Field Equations}

In this subsection we present the solutions of the field equations for the anisotropic cosmological model, considering the temporal evolution of the scale factors with barotropic fluid and standard matter. The solutions obtained already consider the particular choice of the Misner-like transformation discussed lines above. Using the metric (6) and a 
co-moving fluid, equations 2 take the following form

$$
\begin{aligned}
& \frac{\dot{\mathrm{m}}_{1}}{\mathrm{Nm}_{1}} \frac{\dot{\mathrm{m}}_{2}}{\mathrm{Nm}_{2}}+\frac{\dot{\mathrm{m}}_{2}}{\mathrm{Nm}_{2}} \frac{\dot{\mathrm{m}}_{3}}{\mathrm{Nm}_{3}}+\frac{\dot{\mathrm{m}}_{1}}{\mathrm{Nm}_{1}} \frac{\dot{\mathrm{m}}_{3}}{\mathrm{Nm}_{3}}+3\left(\frac{\dot{\eta}}{\mathrm{N} \eta}\right)^{2}-8 \pi \mathrm{G} \rho \\
& -\frac{1}{2}\left(\frac{1}{2} \frac{\dot{\phi}_{1}^{2}}{\mathrm{~N}^{2}}+\mathrm{V}\left(\phi_{1}\right)\right)-\frac{1}{4} \frac{\dot{\phi}_{2}^{2}}{\mathrm{~N}^{2}}=0 \\
& -\frac{\dot{\mathrm{N}}}{\mathrm{N}^{2}}\left[\frac{\dot{\mathrm{m}}_{2}}{\mathrm{Nm}_{2}}+\frac{\dot{\mathrm{m}}_{3}}{\mathrm{Nm}_{3}}+2 \frac{\dot{\eta}}{\mathrm{N} \eta}\right]+\frac{\ddot{\mathrm{m}}_{2}}{\mathrm{~N}^{2} \mathrm{~m}_{2}}+\frac{\ddot{\mathrm{m}}_{3}}{\mathrm{~N}^{2} \mathrm{~m}_{3}}+\frac{\dot{\mathrm{m}}_{2}}{\mathrm{Nm}_{2}} \frac{\dot{\mathrm{m}}_{3}}{\mathrm{Nm}_{3}}+2 \frac{\ddot{\eta}}{\mathrm{N}^{2} \eta} \\
& +\left(\frac{\dot{\eta}}{\mathrm{N} \eta}\right)^{2}+3 \frac{\dot{\eta}}{\mathrm{N} \eta}\left[\frac{\dot{\mathrm{m}}_{2}}{\mathrm{Nm}_{2}}+\frac{\dot{\mathrm{m}}_{3}}{\mathrm{Nm}_{3}}\right]+\frac{1}{2}\left(\frac{1}{2} \frac{\dot{\phi}_{1}^{2}}{\mathrm{~N}^{2}}-\mathrm{V}\left(\phi_{1}\right)\right)+\frac{1}{4} \frac{\dot{\phi}_{2}^{2}}{\mathrm{~N}^{2}}+8 \pi \mathrm{GP}=0, \\
& -\frac{\dot{\mathrm{N}}}{\mathrm{N}^{2}}\left[\frac{\dot{\mathrm{m}}_{1}}{\mathrm{Nm}_{1}}+\frac{\dot{\mathrm{m}}_{3}}{\mathrm{Nm}_{3}}+2 \frac{\dot{\eta}}{\mathrm{N} \eta}\right]+\frac{\ddot{\mathrm{m}}_{1}}{\mathrm{~N}^{2} \mathrm{~m}_{1}}+\frac{\ddot{\mathrm{m}}_{3}}{\mathrm{~N}^{2} \mathrm{~m}_{3}}+\frac{\dot{\mathrm{m}}_{1}}{\mathrm{Nm}_{1}} \frac{\dot{\mathrm{m}}_{3}}{\mathrm{Nm}_{3}}+2 \frac{\ddot{\eta}}{\mathrm{N}^{2} \eta} \\
& +\left(\frac{\dot{\eta}}{\mathrm{N} \eta}\right)^{2}+3 \frac{\dot{\eta}}{\mathrm{N} \eta}\left[\frac{\dot{\mathrm{m}}_{1}}{\mathrm{Nm}_{1}}+\frac{\dot{\mathrm{m}}_{3}}{\mathrm{Nm}_{3}}\right]+\frac{1}{2}\left(\frac{1}{2} \frac{\dot{\phi}_{1}^{2}}{\mathrm{~N}^{2}}-\mathrm{V}\left(\phi_{1}\right)\right)+\frac{1}{4} \frac{\dot{\phi}_{2}^{2}}{\mathrm{~N}^{2}}+8 \pi \mathrm{GP}=0, \\
& -\frac{\dot{\mathrm{N}}}{\mathrm{N}^{2}}\left[\frac{\dot{\mathrm{m}}_{1}}{\mathrm{Nm}_{1}}+\frac{\dot{\mathrm{m}}_{2}}{\mathrm{Nm}_{3}}+2 \frac{\dot{\eta}}{\mathrm{N} \eta}\right]+\frac{\ddot{\mathrm{m}}_{1}}{\mathrm{~N}^{2} \mathrm{~m}_{1}}+\frac{\ddot{\mathrm{m}}_{2}}{\mathrm{~N}^{2} \mathrm{~m}_{2}}+\frac{\dot{\mathrm{m}}_{1}}{\mathrm{Nm}_{1}} \frac{\dot{\mathrm{m}}_{2}}{\mathrm{Nm}_{2}}+2 \frac{\ddot{\eta}}{\mathrm{N}^{2} \eta} \\
& +\left(\frac{\dot{\eta}}{\mathrm{N} \eta}\right)^{2}+3 \frac{\dot{\eta}}{\mathrm{N} \eta}\left[\frac{\dot{\mathrm{m}}_{1}}{\mathrm{Nm}_{1}}+\frac{\dot{\mathrm{m}}_{2}}{\mathrm{Nm}_{2}}\right]+\frac{1}{2}\left(\frac{1}{2} \frac{\dot{\phi}_{1}^{2}}{\mathrm{~N}^{2}}-\mathrm{V}\left(\phi_{1}\right)\right)+\frac{1}{4} \frac{\dot{\phi}_{2}^{2}}{\mathrm{~N}^{2}}+8 \pi \mathrm{GP}=0,
\end{aligned}
$$

equations 9 -12 represent the $\left(\begin{array}{l}0 \\ 0\end{array}\right),\left(\begin{array}{l}1 \\ 1\end{array}\right),\left(\begin{array}{l}2 \\ 2\end{array}\right)$ and the $\left(\begin{array}{l}3 \\ 3\end{array}\right)$ components, respectively and the dot $\left({ }^{\cdot}\right)$ represents a time derivative. The corresponding Klein-Gordon (KG) equations are given by

$$
\begin{aligned}
\frac{\dot{\mathrm{N}}}{\mathrm{N}} \frac{\dot{\phi}_{1}^{2}}{\mathrm{~N}^{2}}-\frac{\dot{\phi}_{1} \ddot{\phi}_{1}}{\mathrm{~N}^{2}}-3 \frac{\dot{\eta}}{\eta} \frac{\dot{\phi}_{1}^{2}}{\mathrm{~N}^{2}}-\dot{\mathrm{V}}=0, & \rightarrow \quad \frac{\mathrm{d}}{\mathrm{dt}} \operatorname{Ln}\left(\frac{\mathrm{N}}{\eta^{3} \dot{\phi}_{1}}\right)=\frac{\mathrm{N}^{2} \dot{\mathrm{V}}}{\dot{\phi}_{1}^{2}} \\
\frac{\dot{\mathrm{N}}}{\mathrm{N}} \frac{\dot{\phi}_{2}^{2}}{\mathrm{~N}^{2}}-\frac{\dot{\phi}_{2} \ddot{\phi}_{2}}{\mathrm{~N}^{2}}-3 \frac{\dot{\eta}}{\eta} \frac{\dot{\phi}_{2}^{2}}{\mathrm{~N}^{2}}=0, & \rightarrow \quad \frac{\mathrm{d}}{\mathrm{dt}} \operatorname{Ln}\left(\frac{\mathrm{N}}{\eta^{3} \dot{\phi}_{2}}\right)=0,
\end{aligned}
$$

where from the last equation in 13 it is easy to see that the solution for the scalar field $\phi_{2}$ (in quadrature form) is given by

$$
\Delta \phi_{2}=\phi_{20} \int \frac{\mathrm{N}}{\eta^{3}} \mathrm{dt}
$$

with $\phi_{20}$ an integration constant.

It is easy to check that if we performed the subtraction of 10 from the component 11 , and identifying that

$$
\frac{1}{\mathrm{~N}}\left[\frac{\dot{\mathrm{m}}_{2}}{\mathrm{Nm}_{2}}-\frac{\dot{\mathrm{m}}_{1}}{\mathrm{Nm}_{1}}\right]^{\bullet}=\frac{1}{\mathrm{~N}^{2}}\left[\frac{\ddot{\mathrm{m}}_{2}}{\mathrm{~m}_{2}}-\frac{\ddot{\mathrm{m}}_{1}}{\mathrm{~m}_{1}}\right]-\frac{1}{\mathrm{~N}^{2}}\left[\left(\frac{\dot{\mathrm{m}}_{2}}{\mathrm{~m}_{2}}\right)^{2}-\left(\frac{\dot{\mathrm{m}}_{1}}{\mathrm{~m}_{1}}\right)^{2}\right]+\frac{\dot{\mathrm{N}}}{\mathrm{N}^{3}}\left[\frac{\dot{\mathrm{m}}_{1}}{\mathrm{~m}_{1}}-\frac{\dot{\mathrm{m}}_{2}}{\mathrm{~m}_{2}}\right],
$$

we get

$$
\frac{1}{\mathrm{~N}}\left[\frac{\dot{\mathrm{m}}_{2}}{\mathrm{Nm}_{2}}-\frac{\dot{\mathrm{m}}_{1}}{\mathrm{Nm}_{1}}\right]^{\bullet}+3 \frac{\dot{\eta}}{\mathrm{N} \eta}\left[\frac{\dot{\mathrm{m}}_{2}}{\mathrm{Nm}_{2}}-\frac{\dot{\mathrm{m}}_{1}}{\mathrm{Nm}_{1}}\right]=0
$$

where [ ] also denotes a time derivative. Defining $R_{21}=\left(\dot{\mathrm{m}}_{2} / \mathrm{Nm}_{2}\right)-\left(\dot{\mathrm{m}}_{1} / \mathrm{Nm}_{1}\right)$, equation (16) can be casted as $\left(\dot{\mathrm{R}}_{21} / \mathrm{R}_{21}\right)+(3 \dot{\eta} / \eta)=0$, giving a solution of the form $\mathrm{R}_{21}=\ell_{21} / \eta^{3}\left(\ell_{12}\right.$ is an integration constant). This procedure can be applied to components 10 - 12 arriving at similar expressions, namely, $\mathrm{R}_{32}=\ell_{32} / \eta^{3}$ and $\mathrm{R}_{13}=\ell_{13} / \eta^{3}$, all three integration constants must satisfy $\ell_{21}+\ell_{32}+\ell_{13}=0$. Now, if take $R_{21}, R_{32}$ and $R_{13}$ together with constraints (8), we obtain the following

$$
\frac{\dot{\mathrm{m}}_{2}}{\mathrm{Nm}_{2}}=\frac{\ell_{2}}{\eta^{3}}, \quad \frac{\dot{\mathrm{m}}_{3}}{\mathrm{Nm}_{3}}=\frac{\ell_{3}}{\eta^{3}}, \quad \frac{\dot{\mathrm{m}}_{1}}{\mathrm{Nm}_{1}}=\frac{\ell_{1}}{\eta^{3}}
$$


in this las three equations $\ell_{2}=\left(\ell_{21}-\ell_{32}\right) / 3, \ell_{3}=\left(\ell_{32}-\ell_{13}\right) / 3, \ell_{1}=\left(\ell_{13}-\ell_{21}\right) / 3$ and they must satisfy $\sum_{j=1}^{3} \ell_{j}=0$ (for more information on what was discussed above we refer the reader to Ref. [79]). Now that equations (17) have a more manageable form, the solutions are straightforward, given by

$$
\mathrm{m}_{\mathrm{i}}(\mathrm{t})=\delta_{\mathrm{i}} \operatorname{Exp}\left[\ell_{\mathrm{i}} \int \frac{\mathrm{Ndt}}{\eta^{3}}\right]
$$

where $\Pi_{\mathrm{j}=1}^{3} \delta_{\mathrm{j}}=1$. Setting the gauge $\mathrm{N} \rightarrow \eta^{3}$, the solution becomes

$$
\mathrm{m}_{\mathrm{i}}(\mathrm{t}) \rightarrow \alpha_{\mathrm{i}} \operatorname{Exp}\left[\ell_{\mathrm{i}} \Delta \mathrm{t}\right]
$$

Unfortunately under this approach we could not find analytical solution for $\eta$, because we need to know the solution for the scalar field $\phi_{1}$ (see equation (9p). To be able to reach a solution we are going to resort to the Hamiltonian formalism. To this end, we employ equation (1) and the line element (6), now the Lagrangian density with the scalar potential field $\mathrm{V}\left(\phi_{1}\right)=\mathrm{V}_{1} \mathrm{e}^{-\lambda_{1} \phi_{1}}$ becomes

$$
\mathcal{L}=\eta^{3}\left(\frac{6}{\mathrm{~N}}\left(\frac{\dot{\eta}}{\eta}\right)^{2}-\frac{1}{\mathrm{~N}}\left[\left(\frac{\dot{\mathrm{m}}_{1}}{\mathrm{~m}_{1}}\right)^{2}+\left(\frac{\dot{\mathrm{m}}_{2}}{\mathrm{~m}_{2}}\right)^{2}+\left(\frac{\dot{\mathrm{m}}_{3}}{\mathrm{~m}_{3}}\right)^{2}\right]-\frac{\dot{\phi}_{1}^{2}}{2 \mathrm{~N}}-\frac{\dot{\phi}_{2}{ }^{2}}{2 \mathrm{~N}}+\mathrm{NV}_{1} \mathrm{e}^{-\lambda_{1} \phi_{1}}\right),
$$

where the momenta are

$$
\begin{aligned}
\Pi_{\eta} & =12 \frac{\eta}{\mathrm{N}} \dot{\eta} & \dot{\eta} & =\frac{\mathrm{N}}{12 \eta} \Pi_{\eta}, \\
\Pi_{\phi_{1}} & =-\frac{\eta^{3}}{\mathrm{~N}} \dot{\phi}_{1} & \dot{\phi}_{1} & =-\frac{\mathrm{N}}{\eta^{3}} \Pi_{\phi_{1}} \\
\Pi_{\phi_{2}} & =-\frac{\eta^{3}}{\mathrm{~N}} \dot{\phi}_{2}, & \dot{\phi}_{2} & =-\frac{\mathrm{N}}{\eta^{3}} \Pi_{\phi_{2}}, \\
\Pi_{1} & =-\frac{2 \eta^{3}}{\mathrm{~N}}\left(\frac{\dot{\mathrm{m}}_{1}}{\mathrm{~m}_{1}^{2}}\right), & \dot{\mathrm{m}}_{1} & =-\frac{\mathrm{Nm}_{1}^{2} \Pi_{1}}{2 \eta^{3}}, \\
\Pi_{2} & =-\frac{2 \eta^{3}}{\mathrm{~N}}\left(\frac{\dot{\mathrm{m}}_{2}}{\mathrm{~m}_{2}^{2}}\right), & \dot{\mathrm{m}}_{2} & =-\frac{\mathrm{Nm}_{2}^{2} \Pi_{2}}{2 \eta^{3}}, \\
\Pi_{3} & =-\frac{2 \eta^{3}}{\mathrm{~N}}\left(\frac{\dot{\mathrm{m}}_{3}}{\mathrm{~m}_{3}^{2}}\right), & \dot{\mathrm{m}}_{3} & =-\frac{\mathrm{Nm}_{3}^{2} \Pi_{3}}{2 \eta^{3}},
\end{aligned}
$$

leading to the Hamiltonian density, which takes the form

$$
\mathcal{H}=\frac{1}{24 \eta} \Pi_{\eta}^{2}-\frac{1}{4 \eta^{3}} \mathrm{~m}_{1}^{2} \Pi_{1}^{2}-\frac{1}{4 \eta^{3}} \mathrm{~m}_{2}^{2} \Pi_{2}^{2}-\frac{1}{4 \eta^{3}} \mathrm{~m}_{3}^{2} \Pi_{3}^{2}-\frac{1}{2 \eta^{3}} \Pi_{\phi_{1}}^{2}-\frac{1}{2 \eta^{3}} \Pi_{\phi_{2}}^{2}-\mathrm{V}_{1} \eta^{3} \mathrm{e}^{-\lambda_{1} \phi_{1}} .
$$

Making the transformation $\Pi_{\eta}=\partial \mathrm{S} / \partial \eta$, and $\Pi_{\mathrm{i}}=\partial \mathrm{S} / \partial \mathrm{m}_{\mathrm{i}}$ and choosing $\eta=\mathrm{e}^{\mathrm{u}}$ and $\mathrm{m}_{\mathrm{i}}=\mathrm{e}^{\mathrm{u}_{\mathrm{i}}}$, where $\mathrm{P}_{\mathrm{i}}=\partial \mathrm{S} / \partial \mathrm{u}_{\mathrm{i}}$ and $\pi_{\mathrm{u}}=\partial \mathrm{S} / \partial \mathrm{u}$, the Hamiltonian density becomes

$$
\mathcal{H}=\frac{\mathrm{e}^{-3 \mathrm{u}}}{24}\left[\pi_{\mathrm{u}}^{2}-6 \mathrm{P}_{1}^{2}-6 \mathrm{P}_{2}^{2}-6 \mathrm{P}_{3}^{2}-12 \Pi_{\phi_{1}}^{2}-12 \Pi_{\phi_{2}}^{2}-\mathrm{U}\left(\mathrm{u}, \phi_{1}\right)\right]
$$

where $\mathrm{U}\left(\mathrm{u}, \phi_{1}\right)=24 \mathrm{~V}_{1} \mathrm{e}^{6 \mathrm{u}-\lambda_{1} \phi_{1}}$ is the potential function. In the gauge $\mathrm{N}=24 \mathrm{e}^{3 \mathrm{u}}$, the Hamilton equations are

$$
\begin{aligned}
& \dot{\mathrm{u}}=2 \pi_{\mathrm{u}}, \quad \dot{\phi}_{1}=-24 \Pi_{\phi_{1}}, \quad \dot{\Pi}_{\phi_{1}}=-\lambda_{1} \mathrm{U}, \quad \dot{\mathrm{u}}_{\mathrm{i}}=-12 \mathrm{P}_{\mathrm{i}}, \\
& \dot{\pi}_{\mathrm{u}}=6 \mathrm{U}, \quad \dot{\phi}_{2}=-24 \Pi_{\phi_{2}}, \quad \dot{\Pi}_{\phi_{2}}=0, \quad \dot{\mathrm{P}}_{\mathrm{i}}=0 .
\end{aligned}
$$

From Hamilton equations (24), we can find relations between the scale factor and the scalar fields, which read

$$
\begin{aligned}
& \dot{\phi}_{1}=-24 \Pi_{\phi_{1}}=4 \lambda_{1} \pi_{\mathrm{u}}+24 \mathrm{p}_{\phi_{1}}=2 \lambda_{1} \dot{\mathrm{u}}+24 \mathrm{p}_{\phi_{1}}, \\
& \dot{\phi}_{2}=-24 \Pi_{\phi_{2}}=24 \mathrm{p}_{\phi_{2}}, \\
& \dot{\mathrm{u}}_{\mathrm{i}}=-12 \mathrm{p}_{\mathrm{i}}
\end{aligned}
$$


being $\mathrm{p}_{\phi_{1}}, \mathrm{p}_{\phi_{2}}$ and $\mathrm{p}_{\mathrm{i}}$ integration constants to be determined by suitable conditions. The solutions of equations 25 read

$$
\begin{aligned}
\Delta \phi_{1} & =2 \lambda_{1} \Delta \mathrm{u}+24 \mathrm{p}_{\phi_{1}} \Delta \mathrm{t} \\
\Delta \phi_{2} & =24 \mathrm{p}_{\phi_{2}} \Delta \mathrm{t} \\
\Delta \mathrm{u}_{\mathrm{i}} & =12 \mathrm{p}_{\mathrm{i}} \Delta \mathrm{t} \\
\mathrm{m}_{\mathrm{i}} & =\beta_{\mathrm{i}} \mathrm{e}^{-12 \mathrm{p}_{\mathrm{i}} \Delta \mathrm{t}}
\end{aligned}
$$

where the constants $\mathrm{p}_{\mathrm{i}}$ must fulfil that $\sum_{\mathrm{i}=1}^{3} \mathrm{p}_{\mathrm{i}}=0$ and $\sum_{\mathrm{i}=1}^{3} \mathrm{p}_{\mathrm{i}}^{2}=2\left(\mathrm{p}_{2}^{2}+\mathrm{p}_{2} \mathrm{p}_{3}+\mathrm{p}_{3}^{2}\right)$. Equations 26 29) are expressions similar to the solution found by algebraic manipulation to 13 for the scalar field $\phi_{2}$ and the Einstein equation (19) for the $\mathrm{m}_{\mathrm{i}}$ functions. These expressions are indeed general relations since they satisfy the EKG equations Eqs.(9 13).

On the other hand, taking into account the constraint $\mathcal{H}=0$, we obtain the temporal dependence for $\pi_{\mathrm{u}}(\mathrm{t})$ which allows us to construct a master equation:

$$
\frac{\mathrm{d} \pi_{\mathrm{u}}}{\alpha_{1} \pi_{\mathrm{u}}^{2}-\alpha_{2} \pi_{\mathrm{u}}-\alpha_{3}}=\mathrm{dt}
$$

where the parameters $\alpha_{\mathrm{i}}$ with $i=1,2,3$, are

$$
\alpha_{1}=2\left(3-\lambda_{1}^{2}\right)=2 \beta, \quad \alpha_{2}=24 \lambda_{1} \mathrm{p}_{\phi_{1}}, \quad \alpha_{3}=72\left[\mathrm{p}_{\phi_{1}}^{2}+\mathrm{c}^{2}\right],
$$

where $c^{2}=p_{\phi_{2}}^{2}+p_{2}^{2}+p_{2} p_{3}+p_{3}^{2}$. In the next subsections, we present solutions for three different values of the parameter $\lambda_{1}$ and also we are able to construct the anisotropic parameters.

\section{B. Case $\alpha_{1}>0$ and $\lambda_{1}<\sqrt{3}$}

For this case, we have that the solution for $\pi_{\mathrm{u}}(\mathrm{t})$ is given by

$$
\pi_{\mathrm{u}}=\frac{1}{4 \beta}\left[\alpha_{2}-\alpha \operatorname{Coth}\left(\frac{\alpha}{2} \mathrm{t}\right)\right]
$$

where $\alpha=24 \omega_{1}$ with $\omega_{1}=\sqrt{3 p_{\phi_{1}}^{2}+c^{2} \beta}$. The solutions of the set of variables $\left(\mathrm{u}, \mathrm{u}_{\mathrm{i}}, \phi_{1}, \phi_{2}\right)$ and $\left(\Pi_{\phi_{1}}, \Pi_{\phi_{2}}, \mathrm{P}_{\mathrm{i}}\right)$ are:

$$
\begin{aligned}
& \mathrm{u}=\mathrm{u}_{0}+12 \frac{\lambda_{1} \mathrm{p}_{\phi_{1}}}{\beta} \mathrm{t}+\ln \left[\operatorname{Csch}\left(12 \omega_{1} \mathrm{t}\right)\right]^{1 / \beta}, \\
& \phi_{1}=\mathrm{p}_{\phi_{1_{0}}}+72 \frac{\mathrm{p}_{\phi_{1}}}{\beta} \mathrm{t}-\operatorname{Ln}\left[\operatorname{Sinh}\left(12 \omega_{1} \mathrm{t}\right)\right]^{2 \lambda_{1} / \beta} \\
& \phi_{2}=\mathrm{p}_{\phi_{2_{0}}}+24 \mathrm{p}_{\phi_{2}} \mathrm{t} \\
& \mathrm{u}_{\mathrm{i}}=-12 \mathrm{p}_{\mathrm{i}} \Delta \mathrm{t} \\
& \Pi_{\phi_{1}}=-3 \frac{\mathrm{p}_{\phi_{1}}}{\beta}+\frac{\lambda_{1} \alpha}{24 \beta} \operatorname{coth}\left(\frac{\alpha}{2} \mathrm{t}\right) \\
& \Pi_{\phi_{2}}=-\mathrm{p}_{\pi_{2}} \\
& \mathrm{P}_{\mathrm{i}}=\mathrm{p}_{\mathrm{i}}
\end{aligned}
$$

here $\left(\mathrm{u}_{0}, \mathrm{p}_{\phi_{1_{0}}}, \mathrm{p}_{\phi_{2_{0}}}, \mathrm{p}_{\mathrm{i}}\right)$ are integration constants. Finally the scale factor $\eta=\mathrm{e}^{\mathrm{u}}$ and the anisotropic parameters take the form

$$
\begin{aligned}
\eta & =\eta_{0} \operatorname{Exp}\left[12 \frac{\lambda_{1} \mathrm{p}_{\phi_{1}}}{\beta} \mathrm{t}\right] \operatorname{Csch}^{\frac{1}{\beta}}\left(12 \sqrt{3 \mathrm{p}_{\phi_{1}}^{2}+\mathrm{p}_{\phi_{2}}^{2} \beta} \mathrm{t}\right), \\
\mathrm{m}_{\mathrm{i}}(\mathrm{t}) & =\beta_{\mathrm{i}} \operatorname{Exp}\left[-12 \mathrm{p}_{\mathrm{i}} \Delta \mathrm{t}\right],
\end{aligned}
$$

where $\eta_{0}=\mathrm{e}^{\mathrm{u}_{0}}, \sum_{\mathrm{i}=1}^{3} \mathrm{p}_{\mathrm{i}}=0$ and $\Pi_{i=1}^{3} \beta_{i}=1$. 


\section{Case $\alpha_{1}<0$ and $\lambda_{1}>\sqrt{3}$}

In this instance its appropriate to take the relation between the momenta

$$
\Pi_{\phi_{1}}=-\frac{\lambda_{1}}{6} \pi_{\mathrm{u}}+\mathrm{p}_{\phi_{1}}, \quad \text { and } \quad \Pi_{\phi_{2}}=-\mathrm{p}_{\phi_{2}}=\text { constant }
$$

then the constant $-\alpha_{2}=24 \lambda_{1} \mathrm{p}_{\phi_{1}}$, allowing us to obtain the temporal dependence for $\pi_{\mathrm{u}}(\mathrm{t})$ with which a master equation can be constructed

$$
\frac{\mathrm{d} \pi_{\mathrm{u}}}{-\alpha_{1} \pi_{\mathrm{u}}^{2}+\alpha_{2} \pi_{\mathrm{u}}-\alpha_{3}}=\mathrm{dt}
$$

where we have included the minus sign such the constant $\alpha_{1}=2\left(\lambda_{1}^{2}-3\right)=2 \beta>0$. Then, defining $\omega_{1}^{2}=\alpha_{2}^{2}-8 \beta \alpha_{3}=$ $576 \omega_{2}^{2}$ with $\omega_{2}^{2}=3 \mathrm{p}_{\phi_{1}}^{2}-\left(\lambda_{1}^{2}-3\right) \mathrm{c}^{2}$, we can rewrite 42 as

$$
\frac{8 \beta d \pi_{u}}{\omega_{1}^{2}-\left(4 \beta \pi_{u}-24 \lambda_{1} \mathrm{p}_{\phi_{1}}\right)^{2}}=\mathrm{dt}
$$

where the constraint over the parameters $\mathrm{p}_{\phi_{1}}>\mathrm{c} \sqrt{\left(\frac{\lambda_{1}}{\sqrt{3}}\right)^{2}-1}$ must be satisfied. In order to be able to integrate 433 , as a final step, we resort to the change of variables $\mathrm{z}=4 \beta \pi_{\mathrm{u}}-24 \lambda_{1} \mathrm{p}_{\phi_{1}}$, thus, the solution for the momenta $\pi_{\mathrm{u}}(\mathrm{t})$ becomes

$$
\pi_{\mathrm{u}}=\frac{6 \lambda_{1} \mathrm{p}_{\phi_{1}}}{\beta}+\frac{6 \omega_{2}}{\beta} \operatorname{Tanh}\left(12 \omega_{2}\left(\mathrm{t}-\mathrm{t}_{0}\right)\right)
$$

Using the relations from (24) and after some algebra, the solutions for the set of variables $\left(\mathrm{u}, \phi_{1}, \phi_{2}\right)$ and $\left(\Pi_{\phi_{1}}, \Pi_{\phi_{2}}\right)$ are:

$$
\begin{aligned}
& \mathrm{u}=\mathrm{u}_{0}+\frac{12 \lambda_{1} \mathrm{p}_{\phi_{1}}}{\beta}\left(\mathrm{t}-\mathrm{t}_{0}\right)+\frac{1}{\beta} \operatorname{Ln}\left[\operatorname{Cosh}\left(12 \omega_{2}\left(\mathrm{t}-\mathrm{t}_{0}\right)\right)\right], \\
& \mathrm{u}_{\mathrm{i}}=-12 \mathrm{p}_{\mathrm{i}} \Delta \mathrm{t}, \\
& \phi_{1}=\phi_{1_{0}}+72 \frac{\mathrm{p}_{\phi_{1}}}{\beta}\left(\mathrm{t}-\mathrm{t}_{0}\right)+\frac{2 \lambda_{1}}{\beta} \operatorname{Ln}\left[\operatorname{Cosh}\left(12 \omega_{2}\left(\mathrm{t}-\mathrm{t}_{0}\right)\right)\right], \\
& \phi_{2}=\phi_{2_{0}}+24 \mathrm{p}_{\phi_{2}}\left(\mathrm{t}-\mathrm{t}_{0}\right), \\
& \Pi_{\phi_{1}}=-\frac{3 \mathrm{p}_{\phi_{1}}}{\beta}-\frac{\lambda_{1} \omega_{2}}{\beta} \operatorname{Tanh}\left(12 \omega_{2}\left(\mathrm{t}-\mathrm{t}_{0}\right)\right), \\
& \Pi_{\phi_{2}}=-\mathrm{p}_{\phi_{2}},
\end{aligned}
$$

where $\left(\mathrm{u}_{0}, \phi_{1_{0}}, \phi_{2_{0}}, \mathrm{p}_{\mathrm{i}}\right)$ are all integration constants. Finally the scale factor becomes

$$
\eta=\eta_{0} \operatorname{Exp}\left[\frac{12 \lambda_{1} \mathrm{p}_{\phi_{1}}}{\beta}\left(\mathrm{t}-\mathrm{t}_{0}\right)\right] \operatorname{Cosh}^{\frac{1}{\beta}}\left(12 \omega_{2}\left(\mathrm{t}-\mathrm{t}_{0}\right)\right),
$$

with $\eta_{0}=\mathrm{e}^{\mathrm{u}_{0}}$ and the anisotropic dimensionless function is

$$
\mathrm{m}_{\mathrm{i}}(\mathrm{t})=\beta_{\mathrm{i}} \operatorname{Exp}\left[-12 \mathrm{p}_{\mathrm{i}} \Delta \mathrm{t}\right]
$$

we can see that equation 52 has the same functional form as before (equation 40 ).

\section{Case $\alpha_{1}=0$ and $\lambda_{1}^{2}=3$.}

For this case the coefficient $\alpha_{1}=0$ and the master equation to solve is reduced to

$$
\int \frac{\mathrm{d} \pi_{\mathrm{u}}}{\alpha_{2} \pi_{\mathrm{u}}-\alpha_{3}}=\int \mathrm{dt}
$$


thus the solution for $\pi_{\mathrm{u}}(\mathrm{t})$ can be obtained relatively easily, which read

$$
\pi_{\mathrm{u}}(\mathrm{t})=\frac{\alpha_{3}}{\alpha_{2}}+\mathrm{pe}^{\alpha_{2}\left(\mathrm{t}-\mathrm{t}_{0}\right)}
$$

where $\mathrm{p}$ is an integration constant. As before, we can use relations from Eq. 24 and after some manipulation, the solutions for $\left(\mathrm{u}, \phi_{1}, \phi_{2}\right)$ and $\left(\Pi_{\phi_{1}}, \Pi_{\phi_{2}}\right)$ are:

$$
\begin{aligned}
& \mathrm{u}=\mathrm{u}_{0}+2 \sqrt{3} \frac{\mathrm{p}_{\phi_{1}}^{2}+\mathrm{c}^{2}}{\mathrm{p}_{\phi_{1}}}\left(\mathrm{t}-\mathrm{t}_{0}\right)+\frac{\sqrt{3} \mathrm{p}}{36 \mathrm{p}_{\phi_{1}}} \mathrm{e}^{24 \sqrt{3} \mathrm{p}_{\phi_{1}}\left(\mathrm{t}-\mathrm{t}_{0}\right)} \\
& \mathrm{u}_{\mathrm{i}}=-12 \mathrm{p}_{\mathrm{i}} \Delta \mathrm{t} \\
& \phi_{1}=\phi_{1_{0}}+12 \frac{\mathrm{c}^{2}-\mathrm{p}_{\phi_{1}}^{2}+}{\mathrm{p}_{\phi_{1}}}\left(\mathrm{t}-\mathrm{t}_{0}\right)+\frac{\mathrm{p}}{6} \mathrm{e}^{24 \sqrt{3} \mathrm{p}_{\phi_{1}}\left(\mathrm{t}-\mathrm{t}_{0}\right)} \\
& \phi_{2}=\phi_{2_{0}}+24 \mathrm{p}_{\phi_{2}}\left(\mathrm{t}-\mathrm{t}_{0}\right) \\
& \Pi_{\phi_{1}}=\frac{1}{2} \frac{\mathrm{p}_{\phi_{1}}^{2}-\mathrm{c}^{2}+}{\mathrm{p}_{\phi_{1}}}-\frac{\sqrt{3} \mathrm{p}}{6} \mathrm{e}^{24 \sqrt{3} \mathrm{p}_{\phi_{1}}\left(\mathrm{t}-\mathrm{t}_{0}\right)} \\
& \Pi_{\phi_{2}}=-\mathrm{p}_{\phi_{2}}
\end{aligned}
$$

again $\left(\mathrm{u}_{0}, \phi_{1_{0}}, \phi_{2_{0}}, \mathrm{p}_{\mathrm{i}}\right)$ are all integration constants. Finally the scale factor $\eta(\mathrm{t})$ for this case is

$$
\eta=\eta_{0} \operatorname{Exp}\left[2 \sqrt{3} \frac{\mathrm{p}_{\phi_{1}}^{2}+\mathrm{c}^{2}}{\mathrm{p}_{\phi_{1}}}\left(\mathrm{t}-\mathrm{t}_{0}\right)\right] \operatorname{Exp}\left[\frac{\sqrt{3} \mathrm{p}}{36 \mathrm{p}_{\phi_{1}}} \mathrm{e}^{24 \sqrt{3} \mathrm{p}_{\phi_{1}}\left(\mathrm{t}-\mathrm{t}_{0}\right)}\right]
$$

where $\eta_{0}=\mathrm{e}^{\mathrm{u}_{0}}$, and as before, the anisotropic dimensionless function $\mathrm{m}_{\mathrm{i}}(\mathrm{t})$ is the same as in (40).

\section{E. Anisotropic Parameters}

In anisotropic cosmology, the Hubble parameter $\mathrm{H}$ is defined in analogy with the FRW cosmology, that is

$$
\mathrm{H}=\frac{\dot{\mathrm{a}}}{\mathrm{a}}=\frac{\dot{\eta}}{\eta}=\frac{1}{3}\left(\mathrm{H}_{\mathrm{x}}+\mathrm{H}_{\mathrm{y}}+\mathrm{H}_{\mathrm{z}}\right)
$$

where $\mathrm{H}_{\mathrm{x}}=\dot{\mathrm{a}}_{1} / \mathrm{a}_{1}, \mathrm{H}_{\mathrm{y}}=\dot{\mathrm{a}}_{2} / \mathrm{a}_{2}$, and $\mathrm{H}_{\mathrm{z}}=\dot{\mathrm{a}}_{3} / \mathrm{a}_{3}$.

The scalar expansion $\theta$, the shear scalar $\sigma^{2}$ and the average anisotropic parameter $\overline{\mathrm{A}}_{\mathrm{m}}$ are defined as

$$
\theta=\sum_{\mathrm{i}=1}^{3} \frac{\dot{\mathrm{a}}_{\mathrm{i}}}{\mathrm{a}_{\mathrm{i}}}=3 \mathrm{H}, \quad \sigma^{2}=\frac{1}{2}\left(\sum_{\mathrm{i}=1}^{3} \mathrm{H}_{\mathrm{i}}^{2}-\frac{1}{3} \theta^{2}\right), \quad \overline{\mathrm{A}}_{\mathrm{m}}=\frac{1}{3} \sum_{\mathrm{i}=1}^{3}\left(\frac{\mathrm{H}_{\mathrm{i}}-\mathrm{H}}{\mathrm{H}}\right)^{2},
$$

respectively.

Following Ref. [81, we consider the volume deceleration parameter,

$$
\mathrm{q}(\mathrm{t})=-\frac{\mathrm{v} \ddot{\mathrm{v}}}{\dot{\mathrm{v}}^{2}}
$$

where $\mathrm{v}=\eta^{3}=\mathrm{a}_{1} \mathrm{a}_{2} \mathrm{a}_{3}$ is the (isotropic) volume function of the Bianchi type I model, and for this case we will have a deceleration parameter for each of the exact solutions given by parameter $\lambda_{1}$, that is

$$
q(t)= \begin{cases}-1-\frac{1}{3} \frac{\beta \omega_{1}^{2}}{\left(\lambda_{1} \mathrm{p}_{\phi_{1}} \operatorname{Sinh}\left(12 \omega_{1} \Delta \mathrm{t}\right)-\omega_{1} \operatorname{Cosh}\left(12 \omega_{1} \Delta \mathrm{t}\right)\right)^{2}}, & \text { for } \lambda_{1}<\sqrt{3} \\ -1-\frac{1}{3} \frac{\beta \omega_{2}^{2}}{\left(\lambda_{1} \mathrm{p}_{\phi_{1}} \operatorname{Cosh}\left(12 \omega_{1} \Delta \mathrm{t}\right)+\omega_{2} \operatorname{Sinh}\left(12 \omega_{1} \Delta \mathrm{t}\right)\right)^{2}}, & \text { for } \lambda_{1}>\sqrt{3} \\ -1-\frac{4 \sqrt{3} p p_{\phi_{1}}^{3} e^{24 \sqrt{3} p_{\phi_{1}} \Delta t}}{\left(p p_{\phi_{1}} e^{24 \sqrt{3} p_{\phi_{1}} \Delta t}+\sqrt{3}\left[p_{\phi_{1}}^{2}+c^{2}\right]\right)^{2}}, & \text { for } \lambda_{1}=\sqrt{3}\end{cases}
$$




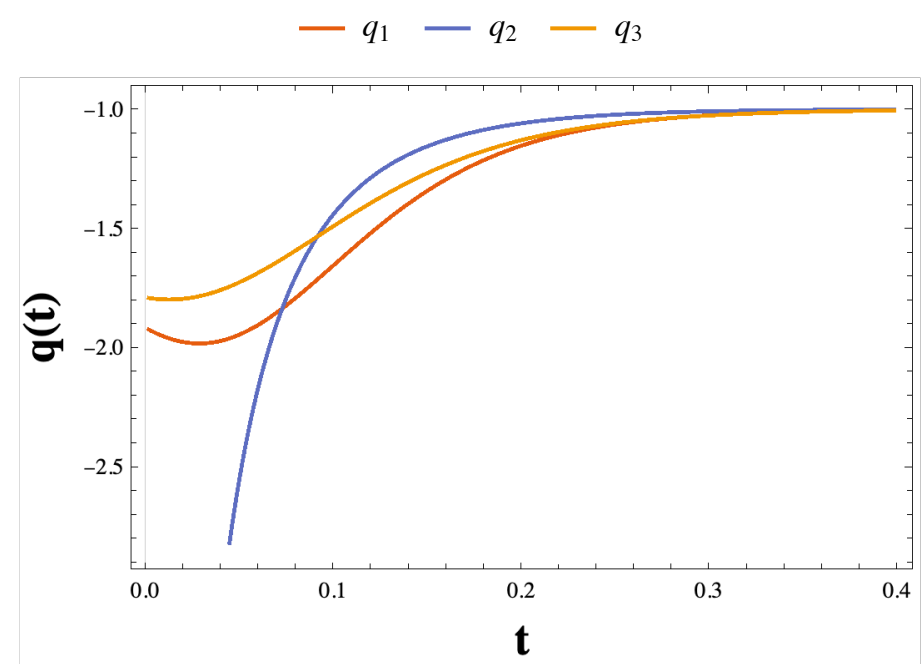

Figure 1: Deceleration parameter for the three classical solutions. Here we have taken $\lambda_{1}=0.5, \lambda_{1}^{\prime}=2, \mathrm{p}_{\phi_{1}}=0.4$, $\mathrm{p}_{\phi_{2}}=0.2, p_{2}=p_{3}=0.01$ and $\mathrm{p}=0.7$.

these observations indicate that the universe presents a volume accelerated expansion in the inflationary epoch. Fig.(1) shows the temporal evolution of the deceleration parameter, where $q_{1}, q_{2}$ and $q_{3}$ stand for the solutions $\lambda_{1}<\sqrt{3}$, $\lambda_{1}>\sqrt{3}, \lambda_{1}=\sqrt{3}$, respectively. Using the results for the average scale factor $\eta$ and the dimensionless anisotropic functions $m_{i}$, the average anisotropic parameter is

$$
\overline{\mathrm{A}}_{\mathrm{m}}= \begin{cases}\frac{8}{3} \frac{\beta^{2}\left(\ell_{2}^{2}+\ell_{2} \ell_{3}+\ell_{3}^{2}\right) \operatorname{Cosh}^{2}\left(12 \omega_{1} \Delta \mathrm{t}\right)}{\left(\omega_{1} \operatorname{Cosh}\left(12 \omega_{1} \Delta \mathrm{t}\right)-\lambda_{1} \mathrm{p}_{\phi_{1}} \operatorname{Sinh}\left(12 \omega_{1} \Delta \mathrm{t}\right)\right)^{2}}, & \text { for } \lambda_{1}<\sqrt{3} \\ \frac{8}{3} \frac{\beta^{2}\left(\ell_{2}^{2}+\ell_{2} \ell_{3}+\ell_{3}^{2}\right) \operatorname{Cosh}^{2}\left(12 \omega_{2} \Delta \mathrm{t}\right)}{\left(\omega_{2} \operatorname{Sinh}\left(12 \omega_{2} \Delta \mathrm{t}\right)+\lambda_{1} \mathrm{p}_{\phi_{1}} \operatorname{Cosh}\left(12 \omega_{2} \Delta \mathrm{t}\right)\right)^{2}}, & \text { for } \lambda_{1}>\sqrt{3} \\ \frac{96 p_{\phi_{1}}^{2}\left(\ell_{2}^{2}+\ell_{2} \ell_{3}+\ell_{3}^{2}\right)}{\left(p p_{\phi_{1}} e^{24 \sqrt{3} p_{\phi_{1}} \Delta t}+\sqrt{3}\left[p_{\phi_{1}}^{2}+c^{2}\right]\right)^{2}} . & \text { for } \lambda_{1}=\sqrt{3}\end{cases}
$$

The other two parameters acquire the form

$$
\begin{aligned}
& \theta= \begin{cases}\frac{36}{\beta}\left(\lambda_{1} \mathrm{p}_{\phi_{1}}-\omega_{1} \operatorname{Ctgh}\left(12 \omega_{1} \Delta \mathrm{t}\right)\right), & \text { for } \lambda_{1}<\sqrt{3}, \\
\frac{36}{\beta}\left[\lambda_{1} \mathrm{p}_{\phi_{1}}+\omega_{2} \operatorname{Tanh}\left(12 \omega_{2} \Delta \mathrm{t}\right)\right], & \text { for } \lambda_{1}>\sqrt{3} \\
6\left(\mathrm{pe}^{24 \sqrt{3} \mathrm{p}_{\phi_{1}} \Delta \mathrm{t}}+\sqrt{3} \frac{\mathrm{p}_{\phi_{1}}^{2}+\mathrm{c}^{2}}{\mathrm{p}_{\phi_{1}}}\right), & \text { for } \lambda_{1}=\sqrt{3}\end{cases} \\
& \sigma^{2}= \begin{cases}648 \frac{\frac{8}{9} \ell^{2} \operatorname{Sinh}^{2}(\gamma)-\left(\omega_{1} \operatorname{Cosh}(\gamma)-\lambda_{1} \mathrm{p}_{\phi_{1}} \operatorname{Sinh}(\gamma)\right)^{2}}{\beta^{2} \operatorname{Sinh}^{2}(\gamma)}, & \text { for } \lambda_{1}<\sqrt{3} \\
648 \frac{\frac{8}{\frac{9}{9} \ell^{2} \operatorname{Cosh}^{2}(\gamma)-\left(\omega_{2} \operatorname{Sinh}(\gamma)+\lambda_{1} \mathrm{p}_{\phi_{1}} \operatorname{Cosh}(\gamma)\right)^{2}}}{\beta^{2} \operatorname{Cosh}^{2}(\gamma)}, & \text { for } \lambda_{1}>\sqrt{3} \\
18\left\{32 \ell^{2}-\left[\frac{\mathrm{p} \mathrm{p}_{\phi_{1}} \mathrm{e}^{24 \sqrt{3} \mathrm{p}_{\phi_{1}} \Delta t}+\sqrt{3}\left(\mathrm{p}_{\phi_{1}}^{2}+\mathrm{c}^{2}\right)}{\mathrm{p}_{\phi_{1}}}\right]^{2}\right\}, & \text { for } \lambda_{1}=\sqrt{3}\end{cases}
\end{aligned}
$$

where we have define $\gamma=12 \omega_{1} \Delta t$ and $\ell^{2}=\ell_{2}^{2}+\ell_{2} \ell_{3}+\ell_{3}^{2}$ strictly for format reasons. In Ref. [82] and references therein, the authors pin down that the red-shift studies place the limit $\sigma / \theta \leq 0.3$ on the ratio of shear $\sigma$ to Hubble 
constant $H$ in the neighborhood of our Galaxy today in order to have a sufficiently isotropic cosmological model, in this regard we obtain

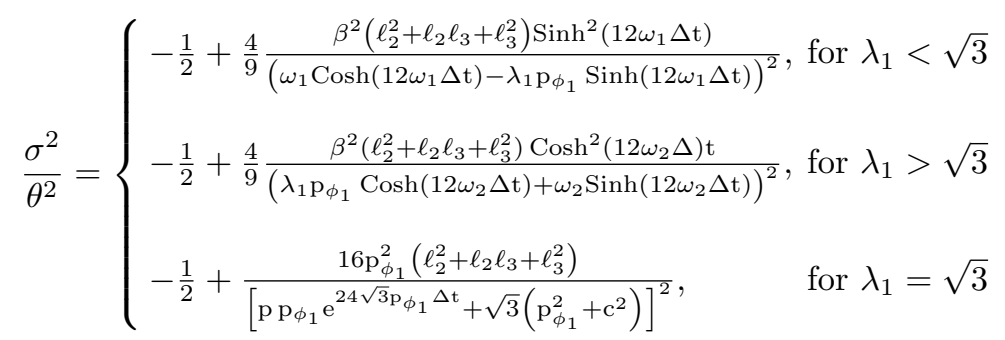

from Eqs. 66 and 69 we can constraint the average anisotropic parameter $\overline{\mathrm{A}}_{\mathrm{m}}$ to the following value for both $\lambda_{1}>\sqrt{3}$ and $\lambda_{1}=\sqrt{3}: \overline{\mathrm{A}}_{\mathrm{m}} \leq 3.54$, signaling that the anisotropic phase still continues.

\section{SECOND MODEL: CHIRAL ANISOTROPIC MODEL}

Now we turn our attention to the second model to be considered. In this case, the action for such a universe is given by

$$
\mathcal{L}=\sqrt{-\mathrm{g}}\left(\mathrm{R}-\frac{1}{2} \mathrm{~g}^{\mu \nu} \mathrm{m}^{\mathrm{ab}} \nabla_{\mu} \phi_{\mathrm{a}} \nabla_{\nu} \phi_{\mathrm{b}}+\mathrm{V}\left(\phi_{1}, \phi_{2}\right)\right),
$$

where $\mathrm{R}$ is the Ricci scalar, $\mathrm{V}\left(\phi_{1}, \phi_{2}\right)=\mathrm{V}_{1} \mathrm{e}^{-\lambda_{1} \phi_{1}}+\mathrm{V}_{2} \mathrm{e}^{-\lambda_{2} \phi_{2}}$ is the corresponding scalar field potential, and $\mathrm{m}^{\mathrm{ab}}$ is a $2 \times 2$ constant matrix and $\mathrm{m}^{12}=\mathrm{m}^{21}$. The EKG equations are obtained varying Eq. 70 with respect to the metric and the scalar fields, resulting in

$$
\begin{gathered}
\mathrm{G}_{\alpha \beta}=-\frac{1}{2} \mathrm{~m}^{\mathrm{ab}}\left(\nabla_{\alpha} \phi_{\mathrm{a}} \nabla_{\beta} \phi_{\mathrm{b}}-\frac{1}{2} \mathrm{~g}_{\alpha \beta} \mathrm{g}^{\mu \nu} \nabla_{\mu} \phi_{\mathrm{a}} \nabla_{\nu} \phi_{\mathrm{b}}\right)+\frac{1}{2} \mathrm{~g}_{\alpha \beta} \mathrm{V}\left(\phi_{1}, \phi_{2}\right), \\
\mathrm{m}^{\mathrm{ab}} \square \phi_{\mathrm{b}}-\frac{\partial \mathrm{V}}{\partial \phi_{\mathrm{a}}}=\mathrm{m}^{\mathrm{ab}} \mathrm{g}^{\mu \nu} \phi_{\mathrm{b}, \mu \nu}-\mathrm{m}^{\mathrm{ab}} \mathrm{g}^{\alpha \beta} \Gamma_{\alpha \beta}^{\nu} \nabla_{\nu} \phi_{\mathrm{b}}-\frac{\partial \mathrm{V}}{\partial \phi_{\mathrm{a}}}=0, \quad \mathrm{a}, \mathrm{b}=1,2 .
\end{gathered}
$$

Consequently the Klein-Gordon equations are

$$
\begin{aligned}
& \mathrm{m}^{11} \phi_{1}^{\prime \prime} \phi_{1}^{\prime}+\mathrm{m}^{12} \phi_{2}^{\prime \prime} \phi_{1}^{\prime}+3 \frac{\eta^{\prime}}{\eta}\left(\mathrm{m}^{11}{\phi_{1}^{\prime}}^{2}+\mathrm{m}^{12} \phi_{1}^{\prime} \phi_{2}^{\prime}\right)+(\dot{\mathrm{V}})_{\phi_{2}}=0 \\
& \mathrm{~m}^{22} \phi_{2}^{\prime \prime} \phi_{2}^{\prime}+\mathrm{m}^{12} \phi_{1}^{\prime \prime} \phi_{2}^{\prime}+3 \frac{\eta^{\prime}}{\eta}\left(\mathrm{m}^{22}{\phi_{2}^{\prime}}^{2}+\mathrm{m}^{12} \phi_{1}^{\prime} \phi_{2}^{\prime}\right)+(\dot{\mathrm{V}})_{\phi_{1}}=0
\end{aligned}
$$

here $\iota=d / d \tau, \mathrm{d} \tau=\mathrm{Ndt}$ and $(\dot{\mathrm{V}})_{\phi_{\mathrm{i}}}$ means that the derivative is calculated maintaining $\phi_{i}$ constant (with $\left.\mathrm{i}=1,2\right)$. An equivalent form to write equations $(73)$ and 74 is

$$
\begin{aligned}
& \mathrm{m}^{11} \dot{\phi}_{1} \frac{\mathrm{d}}{\mathrm{dt}} \operatorname{Ln}\left(\frac{\mathrm{N}}{\eta^{3} \dot{\phi}_{1}}\right)+\mathrm{m}^{12} \dot{\phi}_{2} \frac{\mathrm{d}}{\mathrm{dt}} \operatorname{Ln}\left(\frac{\mathrm{N}}{\eta^{3} \dot{\phi}_{2}}\right)=\frac{\mathrm{N}^{2}(\dot{\mathrm{V}})_{\phi_{2}}}{\dot{\phi}_{1}}, \\
& \mathrm{~m}^{12} \dot{\phi}_{1} \frac{\mathrm{d}}{\mathrm{dt}} \operatorname{Ln}\left(\frac{\mathrm{N}}{\eta^{3} \dot{\phi}_{1}}\right)+\mathrm{m}^{22} \dot{\phi}_{2} \frac{\mathrm{d}}{\mathrm{dt}} \operatorname{Ln}\left(\frac{\mathrm{N}}{\eta^{3} \dot{\phi}_{2}}\right)=\frac{\mathrm{N}^{2}(\dot{\mathrm{V}})_{\phi_{1}}}{\dot{\phi}_{2}} .
\end{aligned}
$$

Taking the metric (5) and pluging it into (70), the Lagrangian density becomes

$$
\begin{aligned}
\mathcal{L}= & \eta^{3}\left(\frac{6}{\mathrm{~N}}\left(\frac{\dot{\eta}}{\eta}\right)^{2}-\frac{1}{\mathrm{~N}}\left[\left(\frac{\dot{\mathrm{m}}_{1}}{\mathrm{~m}_{1}}\right)^{2}+\left(\frac{\dot{\mathrm{m}}_{2}}{\mathrm{~m}_{2}}\right)^{2}+\left(\frac{\dot{\mathrm{m}}_{3}}{\mathrm{~m}_{3}}\right)^{2}\right]\right. \\
& \left.-\mathrm{m}^{11} \frac{\dot{\phi}_{1}^{2}}{2 \mathrm{~N}}-\mathrm{m}^{22} \frac{\dot{\phi}_{2}^{2}}{2 \mathrm{~N}}-\mathrm{m}^{12} \frac{\dot{\phi}_{1} \dot{\phi}_{2}}{\mathrm{~N}}+\mathrm{N}\left[\mathrm{V}_{1} \mathrm{e}^{-\lambda_{1} \phi_{1}}+\mathrm{V}_{2} \mathrm{e}^{-\lambda_{2} \phi_{2}}\right]\right),
\end{aligned}
$$


and the momenta are

$$
\begin{aligned}
\Pi_{\eta} & =12 \frac{\eta}{\mathrm{N}} \dot{\eta}, & \dot{\eta} & =\frac{N}{12 \eta} \Pi_{\eta} \\
\Pi_{\phi_{1}} & =-\frac{\eta^{3}}{\mathrm{~N}}\left(\mathrm{~m}^{11} \dot{\phi}_{1}+\mathrm{m}^{12} \dot{\phi}_{2}\right), & \dot{\phi}_{1} & =\frac{\mathrm{N}}{\eta^{3} \triangle}\left(-\mathrm{m}^{22} \Pi_{\phi_{1}}+\mathrm{m}^{12} \Pi_{\phi_{2}}\right) \\
\Pi_{\phi_{2}} & =-\frac{\eta^{3}}{\mathrm{~N}}\left(\mathrm{~m}^{22} \dot{\phi}_{2}+\mathrm{m}^{12} \dot{\phi}_{1}\right), & \dot{\phi}_{2} & =\frac{N}{\eta^{3} \Delta}\left(m^{12} \Pi_{\phi_{1}}-m^{11} \Pi_{\phi_{2}}\right) \\
\Pi_{1} & =-\frac{2 \eta^{3}}{\mathrm{~N}}\left(\frac{\dot{\mathrm{m}}_{1}}{\mathrm{~m}_{1}^{2}}\right), & \dot{m}_{1} & =-\frac{N m_{1}^{2} \Pi_{1}}{2 \eta^{3}} \\
\Pi_{2} & =-\frac{2 \eta^{3}}{\mathrm{~N}}\left(\frac{\dot{\mathrm{m}}_{2}}{\mathrm{~m}_{2}^{2}}\right), & \dot{m}_{2} & =-\frac{N m_{2}^{2} \Pi_{2}}{2 \eta^{3}} \\
\Pi_{3} & =-\frac{2 \eta^{3}}{\mathrm{~N}}\left(\frac{\dot{\mathrm{m}}_{3}}{\mathrm{~m}_{3}^{2}}\right), & \dot{m}_{3} & =-\frac{N m_{3}^{2} \Pi_{3}}{2 \eta^{3}}
\end{aligned}
$$

where $\triangle=\mathrm{m}^{11} \mathrm{~m}^{22}-\left(\mathrm{m}^{12}\right)^{2}$. Writing 77 in a canonical form, i.e. $\mathcal{L}_{\text {can }}=\Pi_{q} \dot{q}-N \mathcal{H}$, we can perform the variation of this canonical Lagrangian with respect to the lapse function $N, \delta \mathcal{L}_{\text {can }} / \delta N=0$, resulting in the constraint $\mathcal{H}=0$, and making the same transformation as in 23 , the Hamiltonian density results in

$$
\begin{aligned}
\mathcal{H}= & \frac{e^{-3 u}}{24}\left[\Pi_{\mathrm{u}}^{2}-6 \mathrm{P}_{1}^{2}-6 \mathrm{P}_{2}^{2}-6 \mathrm{P}_{3}^{2}-\frac{12 \mathrm{~m}^{22}}{\triangle} \Pi_{\phi_{1}}^{2}-\frac{12 \mathrm{~m}^{11}}{\triangle} \Pi_{\phi_{2}}^{2}+\frac{24 \mathrm{~m}^{12}}{\triangle} \Pi_{\phi_{1}} \Pi_{\phi_{2}}\right. \\
& \left.-24 \mathrm{~V}_{1} \mathrm{e}^{-\lambda_{1} \phi_{1}+6 \mathrm{u}}-24 \mathrm{~V}_{2} \mathrm{e}^{-\lambda_{2} \phi_{2}+6 \mathrm{u}}\right] .
\end{aligned}
$$

Proposing the following canonical transformation on the variables $\left(\eta, \phi_{1}, \phi_{2}, \mathrm{u}_{\mathrm{i}}\right) \leftrightarrow\left(\xi_{1}, \xi_{2}, \xi_{3}, \mathrm{u}_{\mathrm{i}}\right)$

$$
\begin{array}{rlrl}
\xi_{1} & =-6 \mathrm{u}+\lambda_{1} \phi_{1}, \\
\xi_{2} & =-6 \mathrm{u}+\lambda_{2} \phi_{2}, & \mathrm{u} & =\frac{\xi_{1}+\xi_{2}-6 \xi_{3}}{12}, \\
\xi_{3} & =-4 \mathrm{u}+\frac{\lambda_{1}}{6} \phi_{1}+\frac{\lambda_{2}}{6} \phi_{2}, \\
\mathrm{u}_{\mathrm{i}} & =\mathrm{u}_{\mathrm{i}}, & \phi_{1} & =\frac{3 \xi_{1}+\xi_{2}-6 \xi_{3}}{2 \lambda_{1}} \\
& \phi_{2}=\frac{\xi_{1}+3 \xi_{2}-6 \xi_{3}}{2 \lambda_{2}}
\end{array}
$$

and setting the gauge $\mathrm{N}=24 \mathrm{e}^{3 \mathrm{u}}$, allows us to find a new set of conjugate momenta $\left(\pi_{1}, \pi_{2}, \pi_{3}\right)$

$$
\begin{aligned}
\Pi_{\mathrm{u}} & =-6 \pi_{1}-6 \pi_{2}-4 \pi_{3}, \\
\Pi_{\phi_{1}} & =\lambda_{1} \pi_{1}+\frac{\lambda_{1}}{6} \pi_{3}, \\
\Pi_{\phi_{2}} & =\lambda_{2} \pi_{2}+\frac{\lambda_{2}}{6} \pi_{3},
\end{aligned}
$$

which finally leads us to the Hamiltonian density

$$
\begin{aligned}
\mathcal{H}= & 12\left(3-\frac{\lambda_{1}^{2} \mathrm{~m}^{22}}{\triangle}\right) \pi_{1}^{2}+12\left(3-\frac{\lambda_{2}^{2} \mathrm{~m}^{11}}{\triangle}\right) \pi_{2}^{2} \\
& +\left(16+\frac{-\lambda_{1}^{2} m^{22}+2 \lambda_{1} \lambda_{2} m^{12}-\lambda_{2}^{2} m^{11}}{3 \triangle}\right) \pi_{3}^{2} \\
& +12\left[\left(4+\frac{\lambda_{1} \lambda_{2} \mathrm{~m}^{12}-\lambda_{1}^{2} \mathrm{~m}^{22}}{3 \triangle}\right) \pi_{1}+\left(4+\frac{\lambda_{1} \lambda_{2} \mathrm{~m}^{12}-\lambda_{2}^{2} \mathrm{~m}^{11}}{3 \triangle}\right) \pi_{2}\right] \pi_{3} \\
& -6 \mathrm{P}_{1}^{2}-6 \mathrm{P}_{2}^{2}-6 \mathrm{P}_{3}^{2}+24\left(3+\frac{\lambda_{1} \lambda_{2} \mathrm{~m}^{12}}{\triangle}\right) \pi_{1} \pi_{2}-24\left(\mathrm{~V}_{1} \mathrm{e}^{-\xi_{1}}+\mathrm{V}_{2} \mathrm{e}^{-\xi_{2}}\right)
\end{aligned}
$$

the parameter $\triangle$ is the same that was defined after Eqns. (78). The form that the Hamiltonian density 82 acquires after applying the transformation 80 into Eq. 79 will, in the end, allows us to obtain the solutions for this model. 
First, let's compute Hamilton's equations, which read

$$
\begin{aligned}
\dot{\xi}_{1}= & 24\left(3-\frac{\lambda_{1}^{2} \mathrm{~m}^{22}}{\triangle}\right) \pi_{1}+24\left(3+\frac{\lambda_{1} \lambda_{2} \mathrm{~m}_{12}}{\triangle}\right) \pi_{2}+12\left(4+\frac{\lambda_{1} \lambda_{2} \mathrm{~m}^{12}-\lambda_{1}^{2} \mathrm{~m}^{22}}{3 \triangle}\right) \pi_{3}, \\
\dot{\xi}_{2}= & 24\left(3-\frac{\lambda_{2}^{2} \mathrm{~m}^{11}}{\triangle}\right) \pi_{2}+24\left(3+\frac{\lambda_{1} \lambda_{2} \mathrm{~m}_{12}}{\triangle}\right) \pi_{1}+12\left(4+\frac{\lambda_{1} \lambda_{2} \mathrm{~m}^{12}-\lambda_{2}^{2} \mathrm{~m}^{11}}{3 \triangle}\right) \pi_{3}, \\
\dot{\xi}_{3}= & 12\left[\left(4+\frac{\lambda_{1} \lambda_{2} \mathrm{~m}^{12}-\lambda_{1}^{2} \mathrm{~m}^{22}}{3 \triangle}\right) \pi_{1}+\left(4+\frac{\lambda_{1} \lambda_{2} \mathrm{~m}^{12}-\lambda_{2}^{2} \mathrm{~m}^{11}}{3 \triangle}\right) \pi_{2}\right] \\
& +2\left(16+\frac{-\lambda_{1}^{2} m^{22}+2 \lambda_{1} \lambda_{2} m^{12}-\lambda_{2}^{2} m^{11}}{3 \triangle}\right) \pi_{3}, \\
\dot{\pi}_{1}= & -24 \mathrm{~V}_{1} \mathrm{e}^{-\xi_{1}}, \quad \dot{\mathrm{P}}_{\mathrm{i}}=0, \\
\dot{\pi}_{2}= & -24 \mathrm{~V}_{2} \mathrm{e}^{-\xi_{2}}, \quad \dot{\mathrm{u}}_{\mathrm{i}}=-12 \mathrm{P}_{\mathrm{i}}, \\
\dot{\pi}_{3}= & 0,
\end{aligned}
$$

from this last set of equations is straightforward to see that $\pi_{3}=\mathrm{p}_{3}$ and $\mathrm{P}_{\mathrm{i}}=\mathrm{n}_{\mathrm{i}}$ are constants and the solutions to $\mathrm{u}_{\mathrm{i}}=\mathrm{u}_{\mathrm{i}_{0}}-12 \mathrm{n}_{\mathrm{i}} \Delta$ t. Taking the time derivative of the first equation in 83), we obtain

$$
\ddot{\xi}_{1}=-576 \mathrm{~V}_{1}\left(3-\frac{\lambda_{1}^{2} \mathrm{~m}^{22}}{\triangle}\right) \mathrm{e}^{-\xi_{1}}-576 \mathrm{~V}_{2}\left(3+\frac{\lambda_{1} \lambda_{2} \mathrm{~m}^{12}}{\triangle}\right) \mathrm{e}^{-\xi_{2}} .
$$

The main purpose of introducing the transformation 80 was to be able to separate the set of equations arising from the Hamiltonian density 82 . To reach a solution to our problem we set to zero the coefficient that is multiplying the mixed momenta term in 82 , which sets the following constraint on the matrix element $\mathrm{m}^{12}$

$$
\mathrm{m}^{12}=\frac{\lambda_{1} \lambda_{2}}{6}\left(1 \pm \sqrt{1+36 \frac{\mathrm{m}^{11} \mathrm{~m}^{22}}{\lambda_{1}^{2} \lambda_{2}^{2}}}\right),
$$

the latter implies that the second term in the square root of 85 is a real number, say $\ell=36\left(\mathrm{~m}^{11} \mathrm{~m}^{22} / \lambda_{1}^{2} \lambda_{2}^{2}\right) \in \mathbb{R}^{+}$, giving the same weight to the matrix elements $\mathrm{m}^{11}$ and $\mathrm{m}^{22}$, whose values are $\mathrm{m}^{11}=\frac{1}{6} \sqrt{\ell} \lambda_{1}^{2}$ and $\mathrm{m}^{22}=\frac{1}{6} \sqrt{\ell} \lambda_{2}^{2}$. Here, we are going to distinguish two possible scenarios for $\mathrm{m}^{12}$ as: $\mathrm{m}_{+}^{12}=\frac{1}{6} \lambda_{1} \lambda_{2}(1+\sqrt{1+\ell})>0$ and $\mathrm{m}_{-}^{12}=$ $-\frac{1}{6} \lambda_{1} \lambda_{2}(\sqrt{1+\ell}-1)<0$. This two choices of $\mathrm{m}^{12}$ enables us to have a quintom like case and quintessence like case, respectively. With these two possible values for the matrix element $\mathrm{m}^{12}$ we can see that $\triangle_{+}=-\frac{1}{18} \lambda_{1}^{2} \lambda_{2}^{2}(1+\sqrt{1+\ell})<$ 0 for $\mathrm{m}_{+}^{12}$ and $\triangle_{-}=\frac{1}{18} \lambda_{1}^{2} \lambda_{2}^{2}(\sqrt{1+\ell}-1)>0$ for $\mathrm{m}_{-}^{12}$.

\section{A. Quintom like case}

We begin by analyzing the quintom like case, for which the matrix element $\mathrm{m}_{-}^{12}=-\frac{1}{6}(\sqrt{1+\ell}-1) \lambda_{1} \lambda_{2}$, the Hamiltonian density is rewritten as,

$$
\begin{aligned}
\mathcal{H}= & -\frac{\pi_{1}^{2}}{\mu_{\ell}}-\frac{\pi_{2}^{2}}{\mu_{\ell}}+\left(48-\frac{1}{3 c_{\ell}}\right)\left(\pi_{1}+\pi_{2}\right) \pi_{3}+\left(16-\frac{1}{18 c_{\ell}}\right) \pi_{3}^{2}-6\left(\mathrm{P}_{1}^{2}+\mathrm{P}_{2}^{2}+\mathrm{P}_{3}^{2}\right) \\
& -24 \mathrm{~V}_{1} \mathrm{e}^{\xi_{1}}-24 \mathrm{~V}_{2} \mathrm{e}^{-\xi_{2}}
\end{aligned}
$$


also we have defined the parameters $\mu_{\ell}=\sqrt{\ell} / 36(1+\sqrt{1+\ell}-\sqrt{\ell})$ and $c_{\ell}=\sqrt{\ell} / 36[(1+\sqrt{1+\ell})+\sqrt{\ell}]$. Thus, Hamilton equations for the new simplified coordinates $\xi_{\mathrm{i}}$ are

$$
\begin{aligned}
& \dot{\xi}_{1}=-\frac{2 \pi_{1}}{\mu_{\ell}}+\left(48-\frac{1}{3 \mathrm{c}_{\ell}}\right) \pi_{3}, \\
& \dot{\xi}_{2}=-\frac{2 \pi_{2}}{\mu_{\ell}}+\left(48-\frac{1}{3 \mathrm{c}_{\ell}}\right) \pi_{3}, \\
& \dot{\xi}_{3}=+\left(48-\frac{1}{3 \mathrm{c}_{\ell}}\right)\left(\pi_{1}+\pi_{2}\right)+2\left(16-\frac{1}{18 \mathrm{c}_{\ell}}\right) \pi_{3},
\end{aligned}
$$

the equations for $\dot{\pi}_{\mathrm{i}}$ remain the same as in Eqs. 83). Taking the derivative of the first equation of (87) yields

$$
\ddot{\xi}_{1}=\frac{48 \mathrm{~V}_{1}}{\mu_{\ell}} \mathrm{e}^{-\xi_{1}}
$$

which has a solution of the form

$$
\mathrm{e}^{-\xi_{1}}=\frac{\mu_{\ell} \mathrm{r}_{1}^{2}}{24 \mathrm{~V}_{1}} \operatorname{Sech}^{2}\left(\mathrm{r}_{1} \mathrm{t}-\mathrm{q}_{1}\right)
$$

From 87 we can see that $\dot{\xi}_{2}$ has the same functional structure as $\dot{\xi}_{1}$, therefore its solution will be of the same form as 89 , so we have

$$
\mathrm{e}^{-\xi_{2}}=\frac{\mu_{\ell} \mathrm{r}_{2}^{2}}{24 \mathrm{~V}_{2}} \operatorname{Sech}^{2}\left(\mathrm{r}_{2} \mathrm{t}-\mathrm{q}_{2}\right),
$$

where $r_{i}$ and $q_{i}$ (with $i=1,2$ ) are integration constants, both at Eq. (89) and Eq. (90). Reinserting these solutions into Hamilton's equations for the momenta, we obtain

$$
\begin{aligned}
& \pi_{1}=\alpha_{1}-\mu_{\ell} \mathrm{r}_{1} \operatorname{Tanh}\left(\mathrm{r}_{1} \mathrm{t}-\mathrm{q}_{1}\right), \\
& \pi_{2}=\alpha_{2}-\mu_{\ell} \mathrm{r}_{2} \operatorname{Tanh}\left(\mathrm{r}_{2} \mathrm{t}-\mathrm{q}_{2}\right) .
\end{aligned}
$$

With (91) and 292, it can be easily checked that the Hamiltonian is identically null when

$$
\alpha_{1}=\alpha_{2}=\frac{72 \mu_{\ell}-1}{6} \mathrm{p}_{3}, \quad \mathrm{p}_{3}^{2}=\frac{\mu_{\ell}\left(\mathrm{r}_{1}^{2}+\mathrm{r}_{2}^{2}\right)+6 \mathrm{n}^{2}}{4\left(72 \mu_{\ell}+1\right)}
$$

where $\mathrm{n}^{2}$ belongs to the contribution on the anisotropic functions and is given by $\mathrm{n}^{2}=\mathrm{n}_{1}^{2}+\mathrm{n}_{2}^{2}+\mathrm{n}_{3}^{2}$. Now we are in position write the solutions for the $\xi_{\text {i }}$ coordinates, which read

$$
\begin{aligned}
\xi_{1}= & \beta_{1}+\operatorname{Ln}\left[\operatorname{Cosh}^{2}\left(\mathrm{r}_{1} \mathrm{t}-\mathrm{q}_{1}\right)\right], \\
\xi_{2}= & \beta_{2}+\operatorname{Ln}\left[\operatorname{Cosh}^{2}\left(\mathrm{r}_{2} \mathrm{t}-\mathrm{q}_{2}\right)\right], \\
\xi_{3}= & \beta_{3}+\mathrm{p}_{3}\left[16\left(1+72 \mu_{\ell}\right)-8 \frac{\mu_{\ell}}{\mathrm{c}_{\ell}}\right] \Delta \mathrm{t}-\left(48-\frac{1}{3 \mathrm{c}_{\ell}}\right) \mu_{\ell} \times \\
& \operatorname{Ln}\left[\operatorname{Cosh}\left(\mathrm{r}_{1} \mathrm{t}-\mathrm{q}_{1}\right) \operatorname{Cosh}\left(\mathrm{r}_{2} \mathrm{t}-\mathrm{q}_{2}\right)\right],
\end{aligned}
$$


here the $\beta_{\mathrm{i}}$, (with $\mathrm{i}=1,2,3$ ), terms are constants coming from integration. Applying the inverse canonical transformation we obtain the solutions in the original variables $\left(\eta, \phi_{1}, \phi_{2}\right)$ as

$$
\begin{aligned}
\eta & =\eta_{0}+\frac{1}{12} \operatorname{Ln}\left[\operatorname{Cosh}^{2}\left(\mathrm{r}_{1} \mathrm{t}-\mathrm{q}_{1}\right) \operatorname{Cosh}^{2}\left(\mathrm{r}_{2} \mathrm{t}-\mathrm{q}_{2}\right)\right]-\frac{1}{2} \mathrm{p}_{3}\left[16\left(1+72 \mu_{\ell}\right)-8 \frac{\mu_{\ell}}{\mathrm{c}_{\ell}}\right] \Delta \mathrm{t} \\
& +\frac{1}{2} \mu_{\ell}\left(48-\frac{1}{3 \mathrm{c}_{\ell}}\right) \operatorname{Ln}\left[\operatorname{Cosh}\left(\mathrm{r}_{1} \mathrm{t}-\mathrm{q}_{1}\right) \operatorname{Cosh}\left(\mathrm{r}_{2} \mathrm{t}-\mathrm{q}_{2}\right)\right], \\
\phi_{1} & =\phi_{10}+\frac{1}{2 \lambda_{1}} \operatorname{Ln}\left[\operatorname{Cosh}^{6}\left(\mathrm{r}_{1} \mathrm{t}-\mathrm{q}_{1}\right) \operatorname{Cosh}^{2}\left(\mathrm{r}_{2} \mathrm{t}-\mathrm{q}_{2}\right)\right]-\frac{3}{\lambda_{1}} \mathrm{p}_{3}\left[16\left(1+72 \mu_{\ell}\right)-8 \frac{\mu_{\ell}}{\mathrm{c}_{\ell}}\right] \Delta \mathrm{t} \\
& +\frac{3}{\lambda_{1}} \mu_{\ell}\left(48-\frac{1}{3 \mathrm{c}_{\ell}}\right) \operatorname{Ln}\left[\operatorname{Cosh}\left(\mathrm{r}_{1} \mathrm{t}-\mathrm{q}_{1}\right) \operatorname{Cosh}\left(\mathrm{r}_{2} \mathrm{t}-\mathrm{q}_{2}\right)\right], \\
\phi_{2} & =\phi_{20}+\frac{1}{2 \lambda_{2}} \operatorname{Ln}\left[\operatorname{Cosh}^{2}\left(\mathrm{r}_{1} \mathrm{t}-\mathrm{q}_{1}\right) \operatorname{Cosh}^{6}\left(\mathrm{r}_{2} \mathrm{t}-\mathrm{q}_{2}\right)\right]-\frac{3}{\lambda_{2}} \mathrm{p}_{3}\left[16\left(1+72 \mu_{\ell}\right)-8 \frac{\mu_{\ell}}{\mathrm{c}_{\ell}}\right] \Delta \mathrm{t} \\
& +\frac{3}{\lambda_{2}} \mu_{\ell}\left(48-\frac{1}{3 c_{\ell}}\right) \operatorname{Ln}\left[\operatorname{Cosh}\left(\mathrm{r}_{1} \mathrm{t}-\mathrm{q}_{1}\right) \operatorname{Cosh}\left(\mathrm{r}_{2} \mathrm{t}-\mathrm{q}_{2}\right)\right],
\end{aligned}
$$

where $\eta_{0}, \phi_{10}$ and $\phi_{20}$ are given in terms of the $\beta_{i}$ constants as

$$
\eta_{0}=\frac{\beta_{1}+\beta_{2}-6 \beta_{3}}{12}, \quad \phi_{10}=\frac{3 \beta_{1}+\beta_{2}-6 \beta_{3}}{2 \lambda_{1}}, \quad \phi_{20}=\frac{\beta_{1}+3 \beta_{2}-6 \beta_{3}}{2 \lambda_{2}} .
$$

\section{B. Quintessence like case}

Now we turn our attention to the quintessence like case, for which the matrix element $\mathrm{m}_{+}^{12}=\frac{1}{6}(1+\sqrt{1+\ell}) \lambda_{1} \lambda_{2}$, then the Hamiltonian density describing this quintessence model is rewritten as

$$
\begin{aligned}
\mathcal{H} & =\frac{\pi_{1}^{2}}{\nu_{\ell}}+\frac{\pi_{2}^{2}}{\nu_{\ell}}+\left(48-\frac{1}{3 c_{\ell}}\right)\left(\pi_{1}+\pi_{2}\right) \pi_{3}+\left(16-\frac{1}{18 c_{\ell}}\right) \pi_{3}^{2}-6\left(\mathrm{P}_{1}^{2}+\mathrm{P}_{2}^{2}+\mathrm{P}_{3}^{2}\right) \\
& -24 \mathrm{~V}_{1} \mathrm{e}^{-\xi_{1}}-24 \mathrm{~V}_{2} \mathrm{e}^{-\xi_{2}},
\end{aligned}
$$

here we define the parameter $\nu_{\ell}=\sqrt{\ell} / 36(\sqrt{1+\ell}+\sqrt{\ell}-1)$ and $\mathrm{c}_{\ell}=\sqrt{\ell} / 36(+\sqrt{\ell}+1-\sqrt{1+\ell})$.

From (99) we can calculate Hamilton equations for the phase space spanned by $\left(\xi_{\mathrm{i}}, \pi_{\mathrm{i}}\right)$, given by

$$
\begin{aligned}
& \dot{\xi}_{1}=\frac{2 \pi_{1}}{\nu_{\ell}}+\left(48-\frac{1}{3 \mathrm{c}_{\ell}}\right) \pi_{3}, \\
& \dot{\xi}_{2}=\frac{2 \pi_{2}}{\nu_{\ell}}+\left(48-\frac{1}{3 \mathrm{c}_{\ell}}\right) \pi_{3}, \\
& \dot{\xi}_{3}=\left(48-\frac{1}{3 \mathrm{c}_{\ell}}\right)\left(\pi_{1}+\pi_{2}\right)+2\left(16-\frac{1}{18 \mathrm{c}_{\ell}}\right) \pi_{3}, \\
& \mathrm{P}_{\mathrm{i}}=\mathrm{n}_{\mathrm{i}}=\text { constant }
\end{aligned}
$$

as in the quintom case $\dot{\pi}_{\mathrm{i}}$ remain the same as in Eq. 83. Proceeding in a similar way as in the previous case, we take the derivative of the first equation in 100, obtaining

$$
\ddot{\xi}_{1}=-\frac{48 \mathrm{~V}_{1}}{\nu_{\ell}} \mathrm{e}^{-\xi_{1}}
$$

which the corresponding solution is

$$
\mathrm{e}^{-\xi_{1}}=\frac{\nu_{\ell} \mathrm{r}_{1}^{2}}{24 \mathrm{~V}_{1}} \operatorname{Csch}^{2}\left(\mathrm{r}_{1} \mathrm{t}-\mathrm{q}_{1}\right)
$$

Also in this quintessence like setting, the $\dot{\xi}_{2}$ functional form is the same as $\dot{\xi}_{1}$, indicating that the solution is of the same type as 102, that is

$$
\mathrm{e}^{-\xi_{2}}=\frac{\nu_{\ell} \mathrm{r}_{2}^{2}}{24 \mathrm{~V}_{2}} \operatorname{Csch}^{2}\left(\mathrm{r}_{2} \mathrm{t}-\mathrm{q}_{2}\right)
$$


in Eq. 102 and Eq. 103 the $r_{i}$ and $q_{i}$ (with $i=1,2$ ) are constants coming from integration. With Eq. 102 and Eq. 103 at hand, we can reinsert them into Hamilton equations for the momenta, giving

$$
\begin{aligned}
& \pi_{1}=-\mathrm{a}_{1}+\nu_{\ell} \mathrm{r}_{1} \operatorname{Coth}\left(\mathrm{r}_{1} \mathrm{t}-\mathrm{q}_{1}\right), \\
& \pi_{2}=-\mathrm{a}_{2}+\nu_{\ell} \mathrm{r}_{2} \operatorname{Coth}\left(\mathrm{r}_{2} \mathrm{t}-\mathrm{q}_{2}\right),
\end{aligned}
$$

where it can be easily verify that with this last two equations, the Hamiltonian is identically zero when

$$
\mathrm{a}_{1}=\mathrm{a}_{2}=\frac{72 \nu_{\ell}+1}{6} \mathrm{p}_{3}, \quad \mathrm{p}_{3}^{2}=\frac{\nu_{\ell}\left(\mathrm{r}_{1}^{2}+\mathrm{r}_{2}^{2}\right)+6 \mathrm{n}^{2}}{4\left(72 \nu_{\ell}-1\right)},
$$

where $\mathrm{n}^{2}=\mathrm{n}_{1}^{2}+\mathrm{n}_{2}^{2}+\mathrm{n}_{3}^{2}$. So, the solutions for the $\xi_{\mathrm{i}}$ coordinates become

$$
\begin{aligned}
\xi_{1} & =\beta_{1}+\operatorname{Ln}\left[\operatorname{Sinh}^{2}\left(\mathrm{r}_{1} \mathrm{t}-\mathrm{q}_{1}\right)\right], \\
\xi_{2} & =\beta_{2}+\operatorname{Ln}\left[\operatorname{Sinh}^{2}\left(\mathrm{r}_{2} \mathrm{t}-\mathrm{q}_{2}\right)\right], \\
\xi_{3} & =\beta_{3}-\mathrm{p}_{3}\left[16\left(72 \nu_{\ell}-1\right)-8 \frac{\nu_{\ell}}{\mathrm{c}_{\ell}}\right] \Delta \mathrm{t}+\left(48-\frac{1}{3 \mathrm{c}_{\ell}}\right) \nu_{\ell} \times \\
& \operatorname{Ln}\left[\operatorname{Sinh}\left(\mathrm{r}_{1} \mathrm{t}-\mathrm{q}_{1}\right) \operatorname{Sinh}\left(\mathrm{r}_{2} \mathrm{t}-\mathrm{q}_{2}\right)\right],
\end{aligned}
$$

where $\beta_{\mathrm{i}}$ are integration constants (with $\mathrm{i}=1,2,3$ ). After applying the inverse canonical transformation we get the solutions in terms of the original variables $\left(\Omega, \phi_{1}, \phi_{2}\right)$ as

$$
\begin{aligned}
\eta= & \eta_{0}+\frac{1}{12} \operatorname{Ln}\left[\operatorname{Sinh}^{2}\left(\mathrm{r}_{1} \mathrm{t}-\mathrm{q}_{1}\right) \operatorname{Sinh}^{2}\left(\mathrm{r}_{2} \mathrm{t}-\mathrm{q}_{2}\right)\right]+\frac{1}{2} \mathrm{p}_{3}\left[16\left(72 \nu_{\ell}-1\right)-8 \frac{\nu_{\ell}}{\mathrm{c}_{\ell}}\right] \Delta \mathrm{t} \\
- & \frac{1}{2}\left(48-\frac{1}{3 \mathrm{c}_{\ell}}\right) \nu_{\ell} \operatorname{Ln}\left[\operatorname{Sinh}\left(\mathrm{r}_{1} \mathrm{t}-\mathrm{q}_{1}\right) \operatorname{Sinh}\left(\mathrm{r}_{2} \mathrm{t}-\mathrm{q}_{2}\right)\right], \\
\phi_{1}= & \phi_{10}+\frac{1}{2 \lambda_{1}}\left[\operatorname{Ln}\left[\operatorname{Sinh}^{6}\left(\mathrm{r}_{1} \mathrm{t}-\mathrm{q}_{1}\right) \operatorname{Sinh}^{2}\left(\mathrm{r}_{2} \mathrm{t}-\mathrm{q}_{2}\right)\right]-6\left(48-\frac{1}{3 \mathrm{c}_{\ell}}\right) \nu_{\ell} \times\right. \\
& \left.\operatorname{Ln}\left[\operatorname{Sinh}\left(\mathrm{r}_{1} \mathrm{t}-\mathrm{q}_{1}\right) \operatorname{Sinh}\left(\mathrm{r}_{2} \mathrm{t}-\mathrm{q}_{2}\right)\right]\right]+\frac{3}{\lambda_{1}} \mathrm{p}_{3}\left[16\left(72 \nu_{\ell}-1\right)-8 \frac{\nu_{\ell}}{\mathrm{c}_{\ell}}\right] \Delta \mathrm{t}, \\
\phi_{2}= & \phi_{20}+\frac{1}{2 \lambda_{2}}\left[\operatorname{Ln}\left[\operatorname{Sinh}^{2}\left(\mathrm{r}_{1} \mathrm{t}-\mathrm{q}_{1}\right) \operatorname{Sinh}^{6}\left(\mathrm{r}_{2} \mathrm{t}-\mathrm{q}_{2}\right)\right]-6\left(48-\frac{1}{3 \mathrm{c}_{\ell}}\right) \nu_{\ell} \times\right. \\
& \left.\operatorname{Ln}\left[\operatorname{Sinh}\left(\mathrm{r}_{1} \mathrm{t}-\mathrm{q}_{1}\right) \operatorname{Sinh}\left(\mathrm{r}_{2} \mathrm{t}-\mathrm{q}_{2}\right)\right]\right]+\frac{3}{\lambda_{2}} \mathrm{p}_{3}\left[16\left(72 \nu_{\ell}-1\right)-8 \frac{\nu_{\ell}}{\mathrm{c}_{\ell}}\right] \Delta \mathrm{t},
\end{aligned}
$$

where $\eta_{0}, \phi_{10}$ and $\phi_{20}$ are given in terms of the $\beta_{i}$ constants as

$$
\eta_{0}=\frac{\beta_{1}+\beta_{2}-6 \beta_{3}}{12}, \quad \phi_{10}=\frac{3 \beta_{1}+\beta_{2}-6 \beta_{3}}{2 \lambda_{1}}, \quad \phi_{20}=\frac{\beta_{1}+3 \beta_{2}-6 \beta_{3}}{2 \lambda_{2}} .
$$

It is clear that the standard quintessence model with two scalar fields cannot be reproduced under this approach, because when we set $\mathrm{m}^{12}=0$, this imply that parameter $\ell$ is equal to zero, then, the matrix elements $\mathrm{m}^{11}=\mathrm{m}^{22}$ are zero too, this was the challenge to resolve.

\section{QUANTUM APPROACH}

Works related to the Wheeler-DeWitt (WDW) equation and the problems that tackles are extensive, for example in Ref. [83, the question of what a typical wave function for the universe is, is addressed. In Ref. [84 an excellent summary is presented on quantum cosmology, where the problem of how the universe emerged from big bang singularity can no longer be neglected in the GUT epoch. On the other hand, the best candidates for quantum solutions become 
those that have a damping behavior with respect to the scale factor, since these allow to obtain good classical solutions when using the WKB approximation for any scenario in the evolution of our universe [85, 86.

In this section we present the quantum version of the classical anisotropic cosmological models studied above along with its solutions. Since we already have the classical Hamiltonian density, the quantum counterpart can be obtained making the usual replacement $\Pi_{\mathrm{q}^{\mu}}=-\mathrm{i} \hbar \partial_{\mathrm{q}^{\mu}}$. First we modified the classical Hamiltonian density (23) in order to consider the factor ordering problem between the function $\mathrm{e}^{-3 \Omega}$ and its moment $\pi_{\mathrm{u}}$, introducing the linear term as $\mathrm{e}^{-3 \mathrm{u}} \pi_{\mathrm{u}}^{2} \rightarrow \mathrm{e}^{-3 \mathrm{u}}\left[\pi_{\mathrm{u}}^{2}+\mathrm{Qi} \hbar \pi_{\mathrm{u}}\right]$ where $\mathrm{Q}$ is a real number that measures the ambiguity in the factor ordering.

\section{A. Quantum Anisotropic Quintessence-K-essence Model}

In this section we present the quantum version for the cosmological model studied in Section $\Pi$ We start with the modified Hamiltonian density,

$$
\mathcal{H}=\pi_{\mathrm{u}}^{2}+\mathrm{Qi} \hbar \pi_{\mathrm{u}}-12 \Pi_{\phi_{1}}^{2}-12 \Pi_{\phi_{2}}^{2}-6 \mathrm{P}_{1}^{2}-6 \mathrm{P}_{2}^{2}-6 \mathrm{P}_{3}^{2}-24 \mathrm{~V}_{1} \mathrm{e}^{6 \mathrm{u}-\lambda_{1} \phi_{1}},
$$

in order to obtain the WDW equation, we implement the following change of variables $\left(\mathrm{u}, \phi_{1}, \phi_{2}, \mathrm{u}_{\mathrm{i}}\right) \leftrightarrow\left(\xi_{1}, \xi_{2}, \xi_{3}\right)$

$$
\begin{array}{rlrl}
\xi_{1} & =6 \mathrm{u}-\lambda_{1} \phi_{1}, \\
\xi_{2} & =\mathrm{u}, & \mathrm{u} & =\xi_{2}, \\
\xi_{3} & =\phi_{2}, & \phi_{1} & =\frac{-\xi_{1}+6 \xi_{2}}{\lambda_{1}}, \\
\phi_{2} & =\xi_{3}, \\
\mathrm{u}_{\mathrm{i}} & =\mathrm{u}_{\mathrm{i}},
\end{array}
$$

where $u_{i}$ are the conjugate coordinate to momenta $P_{i}$, and also, obtaining a new set of conjugate momenta (in the same manner as (21)), of the variables $\left(\xi_{1}, \xi_{2}, \xi_{3}\right)$, namely $\left(\pi_{1}, \pi_{2}, \pi_{3}\right)$, which read

$$
\pi_{\mathrm{u}}=6 \pi_{1}+\pi_{2}, \quad \Pi_{\phi_{1}}=-\lambda_{1} \pi_{1}, \quad \Pi_{\phi_{2}}=\pi_{3},
$$

which in turn transforms the Hamiltonian density 112 as

$$
\mathcal{H}=12\left(3-\lambda_{1}^{2}\right) \pi_{1}^{2}+\pi_{2}^{2}+12 \pi_{1} \pi_{2}-12 \pi_{3}^{2}+\mathrm{i} \hbar \mathrm{Q}\left(6 \pi_{1}+\pi_{2}\right)-6 \mathrm{P}_{1}^{2}-6 \mathrm{P}_{2}^{2}-6 \mathrm{P}_{3}^{2}-24 \mathrm{~V}_{1} \mathrm{e}^{\xi_{1}}
$$

Introducing the replacement $\pi_{\mathrm{q}^{\mu}}=-\mathrm{i} \hbar \partial_{\mathrm{q}^{\mu}}$, the WDW equation becomes

$$
\begin{aligned}
\mathcal{H} \Psi= & -12 \hbar^{2}\left(3-\lambda_{1}^{2}\right) \frac{\partial^{2} \Psi}{\partial \xi_{1}^{2}}-\hbar^{2} \frac{\partial^{2} \Psi}{\partial \xi_{2}^{2}}-12 \hbar^{2} \frac{\partial^{2} \Psi}{\partial \xi_{1} \partial \xi_{2}}+12 \hbar^{2} \frac{\partial^{2} \Psi}{\partial \xi_{3}^{2}}+ \\
& Q \hbar^{2}\left(6 \frac{\partial \Psi}{\partial \xi_{1}}+\frac{\partial \Psi}{\xi_{2}}\right)+6 \hbar^{2}\left(\frac{\partial^{2} \Psi}{\partial u_{1}^{2}}+\frac{\partial^{2} \Psi}{\partial u_{2}^{2}}+\frac{\partial^{2} \Psi}{\partial u_{3}^{3}}\right)-24 V_{1} e^{\xi_{1}} \Psi=0
\end{aligned}
$$

due that the scalar potential does not depend on the coordinates $\left(\xi_{2}, \xi_{3}, \mathrm{u}_{\mathrm{i}}\right)$, we propose the following ansatz for the wave function $\Psi\left(\xi_{1}, \xi_{2}, \xi_{3}, \mathrm{u}_{\mathrm{i}}\right)=\mathrm{e}^{-\left(\mathrm{a}_{2} \xi_{2}+\mathrm{a}_{3} \xi_{3}+\mathrm{a}_{4} \mathrm{u}_{1}+\mathrm{a}_{5} \mathrm{u}_{2}+\mathrm{a}_{6} \mathrm{u}_{3}\right) / \hbar} \mathrm{G}\left(\xi_{1}\right)$ where the $\mathrm{a}_{\mathrm{i}}$ are arbitrary constants. Introducing the mentioned ansatz in 116 we have that

$$
-12 \hbar^{2}\left(3-\lambda_{1}^{2}\right) \frac{1}{\mathrm{G}} \frac{\mathrm{d}^{2} \mathrm{G}}{\mathrm{d} \xi_{1}^{2}}+6 \hbar\left(2 \mathrm{a}_{2}+\hbar \mathrm{Q}\right) \frac{1}{\mathrm{G}} \frac{\mathrm{dG}}{\mathrm{d} \xi_{1}}-\mathrm{a}_{2}\left(\mathrm{a}_{2}+\hbar \mathrm{Q}\right)+12 \mathrm{a}_{3}^{2}+6 \mathrm{a}_{0}^{2}-24 \mathrm{~V}_{1} \mathrm{e}^{\xi_{1}}=0,
$$

where $a_{0}^{2}=a_{4}^{2}+a_{5}^{2}+a_{6}^{2}$, and also we have divided the whole equation by the ansatz; this in turn leads us to the following differential equation

$$
\frac{\mathrm{d}^{2} \mathrm{G}}{\mathrm{d} \xi_{1}^{2}}-\frac{2 \mathrm{a}_{2}+\hbar \mathrm{Q}}{2 \hbar\left(3-\lambda_{1}^{2}\right)} \frac{\mathrm{dG}}{\mathrm{d} \xi_{1}}+\frac{1}{12 \hbar^{2}\left(3-\lambda_{1}^{2}\right)}\left[24 \mathrm{~V}_{1} \mathrm{e}^{\xi_{1}}+\eta\right] \mathrm{G}=0
$$


here $\eta=a_{2}\left(a_{2}+\hbar \mathrm{Q}\right)-12 \mathrm{a}_{3}^{2}-6 \mathrm{a}_{0}^{2}$. The last equation can be casted as $\mathrm{y}^{\prime \prime}+\mathrm{ay}^{\prime}+\left(\mathrm{be}{ }^{\kappa \mathrm{x}}+\mathrm{c}\right) \mathrm{y}=0$ (and whose solutions will depend on the value of $\lambda_{1}$ ) [87, where

$$
\mathrm{y}=\operatorname{Exp}\left(-\frac{\mathrm{ax}}{2}\right) \mathrm{Z}_{\nu}\left(\frac{2 \sqrt{\mathrm{b}}}{\kappa} \mathrm{e}^{\frac{\kappa \mathrm{x}}{2}}\right),
$$

here $\mathrm{Z}_{\nu}$ is the Bessel function and $\nu=\sqrt{a^{2}-4 c} / \kappa$ being the order. The corresponding relations between the coefficients of (117) and a, b, c and $\kappa$ are

$$
\begin{gathered}
\mathrm{a}=\left\{\begin{array}{l}
\frac{2 \mathrm{a}_{2}+\hbar \mathrm{Q}}{2 \hbar\left(\lambda_{1}^{2}-3\right)}, \text { when } \lambda_{1}^{2}>3 \\
-\frac{2 \mathrm{a}_{2}+\hbar \mathrm{Q}}{2 \hbar\left(3-\lambda_{1}^{2}\right)}, \text { when } \lambda_{1}^{2}<3
\end{array}\right. \\
\mathrm{b}= \begin{cases}-\frac{2 \mathrm{~V}_{0}}{\hbar^{2}\left(\lambda^{2}-3\right)}, & \text { when } \lambda_{1}^{2}>3 \\
\frac{2 \mathrm{~V}_{0}}{\hbar^{2}\left(3-\lambda^{2}\right)}, & \text { when } \lambda_{1}^{2}<3\end{cases} \\
\mathrm{c}=\left\{\begin{array}{l}
-\frac{\eta}{12 \hbar^{2}\left(\lambda_{1}^{2}-3\right)}, \text { when } \lambda_{1}^{2}>3 \\
\frac{\eta}{12 \hbar^{2}\left(3-\lambda_{1}^{2}\right)}, \text { when } \lambda_{1}^{2}<3
\end{array}\right. \\
\kappa=1,
\end{gathered}
$$

according to the constant $\mathrm{b}$, the solution to the function $\mathrm{G}$ becomes

$$
\begin{aligned}
\mathrm{G}\left(\xi_{1}\right)=\operatorname{Exp}\left(-\frac{2 \mathrm{a}_{2}+\hbar \mathrm{Q}}{4 \hbar\left(\lambda_{1}^{2}-3\right)} \xi_{1}\right) \mathrm{K}_{\nu_{1}}\left(\frac{2}{\hbar} \sqrt{\frac{2 \mathrm{~V}_{0}}{\lambda^{2}-3}} \mathrm{e}^{\frac{\xi_{1}}{2}}\right), & \lambda_{1}^{2}>3 \\
\mathrm{G}\left(\xi_{1}\right)=\operatorname{Exp}\left(\frac{2 \mathrm{a}_{2}+\hbar \mathrm{Q}}{4 \hbar\left(3-\lambda_{1}^{2}\right) \xi_{1}}\right) \quad \mathrm{J}_{\nu_{2}}\left(\frac{2}{\hbar} \sqrt{\frac{2 \mathrm{~V}_{0}}{3-\lambda_{1}^{2}}} \mathrm{e}^{\frac{\xi_{1}}{2}}\right), & \lambda_{1}^{2}<3
\end{aligned}
$$

and the wave function takes the form

$$
\begin{aligned}
\Psi_{\nu_{1}}= & \operatorname{Exp}\left(-\frac{2 \mathrm{a}_{2}+\hbar \mathrm{Q}}{4 \hbar\left(\lambda_{1}^{2}-3\right)} \xi_{1}-\frac{\mathrm{a}_{2} \xi_{2}+\mathrm{a}_{3} \xi_{3}}{\hbar}-\frac{\mathrm{a}_{4} \mathrm{u}_{1}+\mathrm{a}_{4} \mathrm{u}_{2}+\mathrm{a}_{6} \mathrm{u}_{3}}{\hbar}\right) \times \\
& K_{\nu_{1}}\left(\frac{2}{\hbar} \sqrt{\frac{2 V_{0}}{\lambda_{1}^{2}-3}} e^{\frac{\xi_{1}}{2}}\right), \quad \lambda_{1}^{2}>3 \\
\Psi_{\nu_{2}}= & \operatorname{Exp}\left(\frac{2 \mathrm{a}_{2}+\hbar \mathrm{Q}}{4 \hbar\left(3-\lambda_{1}^{2}\right)} \xi_{1}-\frac{\mathrm{a}_{2} \xi_{2}+\mathrm{a}_{3} \xi_{3}}{\hbar}-\frac{\mathrm{a}_{4} \mathrm{u}_{1}+\mathrm{a}_{4} \mathrm{u}_{2}+\mathrm{a}_{6} \mathrm{u}_{3}}{\hbar}\right) \times \\
& J_{\nu_{2}}\left(\frac{2}{\hbar} \sqrt{\frac{2 V_{0}}{3-\lambda^{2}}} e^{\frac{\xi_{1}}{2}}\right), \quad \lambda_{1}^{2}<3 .
\end{aligned}
$$

where $\nu_{1}=\sqrt{\left(-\frac{2 \mathrm{a}_{2}+\hbar \mathrm{Q}}{4 \hbar\left(\lambda_{1}^{2}-3\right)}\right)^{2}+\frac{4 \eta}{12 \hbar^{2}\left(\lambda_{1}^{2}-3\right)}}$ and $\nu_{2}=\sqrt{\left(\frac{2 \mathrm{a}_{2}+\hbar \mathrm{Q}}{4 \hbar\left(3-\lambda_{1}^{2}\right)}\right)^{2}-\frac{4 \eta}{12 \hbar^{2}\left(3-\lambda_{1}^{2}\right)}}$ are the corresponding order of $\Psi$. Applying the inverse transformation on the variables $\xi_{\mathrm{i}}$, we can write the wave function in terms of the original 
variables $\left(\mathrm{A}=\mathrm{e}^{\Omega}, \phi_{\mathrm{i}}, \mathrm{m}_{\mathrm{i}}=\mathrm{e}^{\mathrm{u}_{\mathrm{i}}}\right)$, which read

$$
\begin{aligned}
\Psi_{\nu_{1}}= & \mathrm{m}_{1}^{-\frac{\mathrm{a}_{4}}{\hbar}} \mathrm{m}_{2}^{-\frac{\mathrm{a}_{5}}{\hbar}} \mathrm{m}_{3}^{-\frac{\mathrm{a}_{6}}{\hbar}} \mathrm{A}^{-\alpha_{1}} \operatorname{Exp}\left(\frac{2 \mathrm{a}_{2}+\hbar \mathrm{Q}}{4 \hbar\left(\lambda_{1}^{2}-3\right)} \lambda_{1} \phi_{1}-\frac{\mathrm{a}_{3}}{\hbar} \phi_{2}\right) \times \\
& K_{\nu_{1}}\left(\frac{2}{\hbar} \sqrt{\frac{2 V_{0}}{\lambda_{1}^{2}-3}} A^{3} e^{\frac{\lambda_{1}}{2} \phi_{1}}\right), \quad \lambda_{1}^{2}>3 \\
\Psi_{\nu_{2}}= & \mathrm{m}_{1}^{-\frac{\mathrm{a}_{4}}{\hbar}} \mathrm{m}_{2}^{-\frac{\mathrm{a}_{5}}{\hbar}} \mathrm{m}_{3}^{-\frac{\mathrm{a}_{6}}{\hbar}} \mathrm{A}^{-\alpha_{2}} \operatorname{Exp}\left(-\frac{2 \mathrm{a}_{2}+\hbar \mathrm{Q}}{4 \hbar\left(\lambda_{1}^{2}-3\right)} \lambda_{1} \phi_{1}-\frac{\mathrm{a}_{3}}{\hbar} \phi_{2}\right) \times \\
& J_{\nu_{2}}\left(\frac{2}{\hbar} \sqrt{\frac{2 V_{0}}{3-\lambda^{2}}} A^{3} e^{\frac{\lambda_{1}}{2} \phi_{1}}\right), \quad \lambda_{1}^{2}<3 .
\end{aligned}
$$

with $\alpha_{1}=\frac{1}{\hbar}\left(a_{2}+\frac{3}{2} \frac{2 a_{2}+\hbar Q}{\lambda_{1}^{2}-3}\right)$ and $\alpha_{2}=\frac{1}{\hbar}\left(a_{2}-\frac{3}{2} \frac{2 a_{2}+\hbar Q}{3-\lambda_{1}^{2}}\right)$. In Fig $\sqrt{2}$ we can see the behavior of the probability

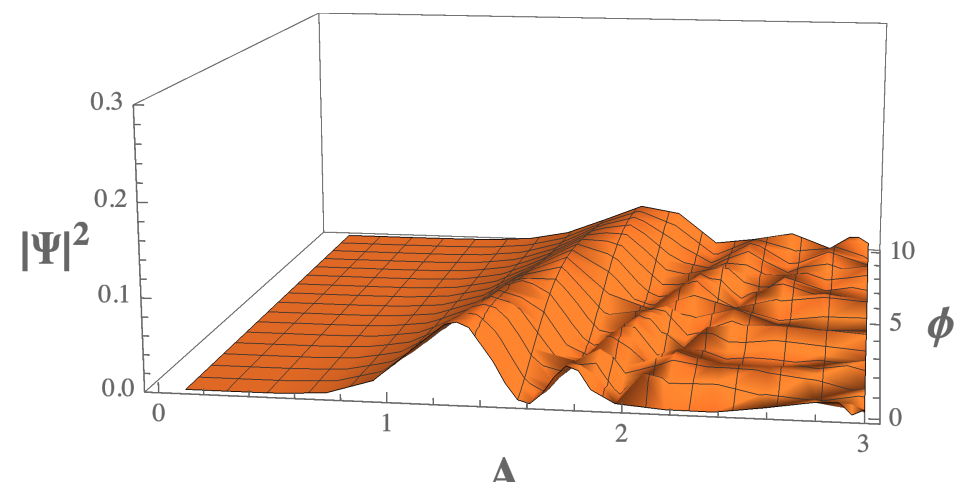

A

Figure 2: Behavior of the probability density for $\lambda_{1}<\sqrt{3}$, for $\mathrm{Q}=1, \lambda_{1}, a_{2}=0.6, a_{3}=1$ and $a_{4}=a_{5}=a_{6}=0.3$.

density of the wave function for the solution $\lambda_{1}<\sqrt{3}$. It is observed that the evolution of the wave function with respect of the scale factor is damped, which is a good characteristic and this kind of behavior also have been reported in $[68,80,88$. In comparison with isotropic model [80, we can see that the anisotropies shrink the probability density of the wave function.
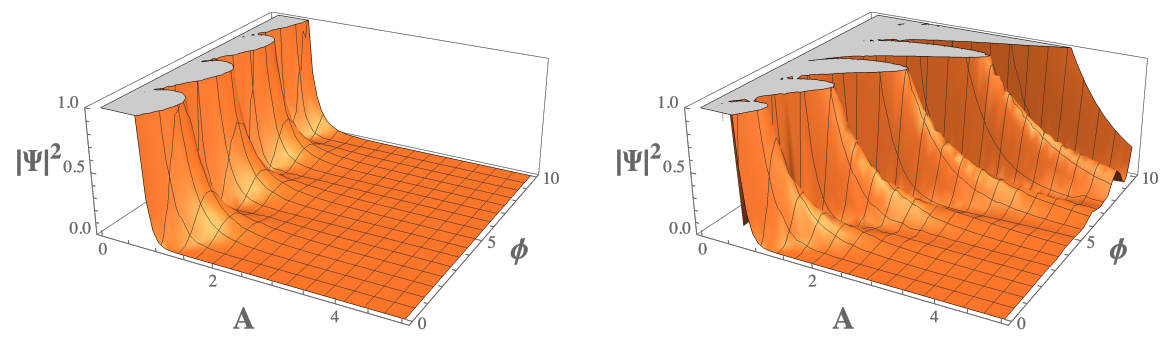

Figure 3: Behavior of the probability density for $\lambda_{1}>\sqrt{3}$. For both figures $\lambda_{1}=6, a_{2}=2, a_{3}=1, a_{4}=a_{5}=a_{6}=0.3$ whereas for the left figure $\mathrm{Q}=-2$ and for the right figure $\mathrm{Q}=-10$.

In Fig. (3) we can observe the evolution of the wave function for the solution $\lambda_{1}>\sqrt{3}$. In this particular case the values of $\mathrm{Q}$ act as a retarder (for negative values) for the wave function and compresses the length over the axis were the scalar field evolves (this should also delay the inflationary epoch), but still having the damped behavior. Contrasting this results with those of the isotropic treatment [80, we can see that anisotropies shrink the the probability density along the evolution of the scalar field. 
Finally, for the particular case of $\lambda_{1}=\sqrt{3}$ the quantum solution for the function $G\left(\xi_{1}\right)$ becomes

$$
\mathrm{G}\left(\xi_{1}\right)=\mathrm{G}_{0} \operatorname{Exp}\left[\frac{\eta}{6 \hbar\left(2 \mathrm{a}_{2}+\hbar \mathrm{Q}\right)} \xi_{1}\right] \operatorname{Exp}\left(\frac{4 \mathrm{~V}_{1}}{\hbar\left(2 \mathrm{a}_{2}+\hbar \mathrm{Q}\right)} \mathrm{e}^{\xi_{1}}\right)
$$

and the wave function is

$$
\begin{aligned}
\Psi\left(\mathrm{A}, \phi_{\mathrm{i}}, \mathrm{m}_{\mathrm{i}}\right)= & \Psi_{0} \mathrm{~m}_{1}^{-\frac{\mathrm{a}_{4}}{\hbar}} \mathrm{m}_{2}^{-\frac{\mathrm{a}_{5}}{\hbar}} \mathrm{m}_{3}^{-\frac{\mathrm{a}_{6}}{\hbar}} \mathrm{A}^{\mathrm{r}} \operatorname{Exp}\left(-\frac{\mathrm{a}_{3}}{\hbar} \phi_{2}-\frac{\lambda_{1}}{6 \hbar\left(2 \mathrm{a}_{2}+\hbar \mathrm{Q}\right)} \phi_{1}\right) \times \\
& \left.\operatorname{Exp}\left[\frac{4 \mathrm{~V}_{1}}{\hbar\left(2 \mathrm{a}_{2}+\hbar \mathrm{Q}\right)} \mathrm{A}^{6} \mathrm{e}^{-\lambda_{1} \phi_{1}}\right)\right] .
\end{aligned}
$$

where the constant $\mathrm{r}=-\frac{\mathrm{a}_{2}}{\hbar}+\frac{\eta}{\hbar\left(2 \mathrm{a}_{2}+\hbar \mathrm{Q}\right)}$.

\section{B. Quantum Anisotropic Quintom Case}

For the second cosmological model, the quintom like case, the quantum version of this model is obtained applying, again, the recipe $\Pi_{\mathrm{q}^{\mu}}=-\mathrm{i} \hbar \partial_{\mathrm{q}^{\mu}}$ to the Hamiltonian density (86), hence

$$
\begin{array}{r}
{\left[\frac{\hbar^{2}}{\mu_{\ell}} \frac{\partial^{2}}{\partial \xi_{1}^{2}}+\frac{\hbar^{2}}{\mu_{\ell}} \frac{\partial^{2}}{\partial \xi_{2}^{2}}-\hbar^{2}\left(48-\frac{1}{3 c_{\ell}}\right)\left(\frac{\partial^{2}}{\partial \xi_{3} \partial \xi_{1}}+\frac{\partial^{2}}{\partial \xi_{3} \partial \xi_{2}}\right)-\hbar^{2}\left(16-\frac{1}{18 c_{\ell}}\right) \frac{\partial^{2}}{\partial \xi_{3}^{2}}\right.} \\
\left.+6 \hbar^{2}\left(\frac{\partial^{2} \Psi}{\partial u_{1}^{2}}+\frac{\partial^{2} \Psi}{\partial u_{2}^{2}}+\frac{\partial^{2} \Psi}{\partial u_{3}^{2}}\right)-24 V_{1} e^{-\xi_{1}}-24 V_{2} e^{-\xi_{2}}\right] \Psi=0
\end{array}
$$

because the scalar potential does not depend on the coordinate $\xi_{3}$, we propose the following ansatz for the wave function $\Psi\left(\xi_{1}, \xi_{2}, \xi_{3}\right)=\mathrm{e}^{\left(\mathrm{a}_{3} \xi_{3}+\mathrm{a}_{4} \mathrm{u}_{1}+\mathrm{a}_{4} \mathrm{u}_{2}+\mathrm{a}_{6} \mathrm{u}_{3}\right) / \hbar} \mathcal{A}\left(\xi_{1}\right) \mathcal{B}\left(\xi_{2}\right)$ where $\mathrm{a}_{\mathrm{i}}$ (with $\left.\mathrm{i}=3,4,5,6\right)$ are an arbitrary constants. Substituting and dividing by the ansatz in 130 , we obtain

$$
\begin{aligned}
\frac{\hbar^{2}}{\mu_{\ell} \mathcal{A}} \frac{\mathrm{d}^{2} \mathcal{A}}{\mathrm{d} \xi_{1}^{2}}+\frac{\hbar^{2}}{\mu_{\ell} \mathcal{B}} \frac{\mathrm{d}^{2} \mathcal{B}}{\mathrm{d} \xi_{2}^{2}} & -a_{3} \hbar\left(48-\frac{1}{3 c_{\ell}}\right)\left(\frac{1}{\mathcal{A}} \frac{d \mathcal{A}}{d \xi_{1}}+\frac{1}{\mathcal{B}} \frac{d \mathcal{B}}{d \xi_{2}}\right) \\
& -a_{3}^{2}\left(16-\frac{1}{18 c_{\ell}}\right)+6 a_{0}^{2}-24 V_{1} e^{-\xi_{1}}-24 V_{2} e^{-\xi_{2}}=0
\end{aligned}
$$

with $\mathrm{a}_{0}^{2}=\mathrm{a}_{4}^{2}+\mathrm{a}_{5}^{2}+\mathrm{a}_{6}^{2}$, where we can separate the equations as

$$
\begin{aligned}
\frac{\mathrm{d}^{2} \mathcal{A}}{\mathrm{d} \xi_{1}^{2}}- & \frac{a_{3} \mu_{\ell}}{\hbar}\left(48-\frac{1}{3 c_{\ell}}\right) \frac{d \mathcal{A}}{d \xi_{1}} \\
& -\frac{\mu_{\ell}}{\hbar^{2}}\left(\frac{a_{3}^{2}}{2}\left(16-\frac{1}{18 c_{\ell}}\right)-3 a_{0}^{2}-\alpha^{2}+24 V_{1} e^{-\xi_{1}}\right) \mathcal{A}=0 \\
\frac{\mathrm{d}^{2} \mathcal{B}}{\mathrm{d} \xi_{2}^{2}}- & \frac{a_{3} \mu_{\ell}}{\hbar}\left(48-\frac{1}{3 c_{\ell}}\right) \frac{d \mathcal{B}}{d \xi_{2}} \\
& -\frac{\mu_{\ell}}{\hbar^{2}}\left(\frac{a_{3}^{2}}{2}\left(16-\frac{1}{18 c_{\ell}}\right)-3 a_{0}^{2}+\alpha^{2}+24 V_{2} e^{-\xi_{2}}\right) \mathcal{B}=0
\end{aligned}
$$

with $\alpha^{2}$ being the separation constant. The corresponding solutions of Eqs. 132 and 133 have the following form 87

$$
\mathrm{Y}(\mathrm{x})=\operatorname{Exp}\left(-\frac{\mathrm{ax}}{2}\right) \mathrm{Z}_{\nu}\left(\frac{2 \sqrt{\mathrm{b}}}{\lambda} \mathrm{e}^{\frac{\lambda x}{2}}\right)
$$


here $\mathrm{Z}_{\nu}$ are the generic Bessel function with order $\nu=\sqrt{\mathrm{a}^{2}-4 \mathrm{c}} / \lambda$. If $\sqrt{b}$ is real, $\mathrm{Z}_{\nu}$ are the ordinary Bessel function, otherwise the solution will be given by the modified Bessel function. Making the following identifications

$$
\begin{aligned}
\lambda & =-1, \\
\mathrm{a} & =-\frac{a_{3} \mu_{\ell}}{\hbar}\left(48-\frac{1}{3 c_{\ell}}\right), \\
\mathrm{b}_{1,2} & =-\frac{\mu_{\ell}}{\hbar^{2}} 24 V_{1,2}, \\
\mathrm{c}_{\mp} & =-\frac{\mu_{\ell}}{\hbar^{2}}\left(a_{3}^{2}\left(8-\frac{1}{36 c_{\ell}}\right)-3 a_{0}^{2} \mp \alpha^{2}\right), \\
\nu_{\mp} & =\sqrt{\frac{\mathrm{a}^{2}}{\mu_{\ell}}+4 \mathrm{c}_{\mp},}
\end{aligned}
$$

we can check that the value for $\sqrt{b}$ is imaginary, which as already mentioned, gives a solution in terms of the modified Bessel function $\mathrm{Z}_{\nu}=\mathrm{K}_{\nu}$ whose order lies in the reals. Thus, the wave function is

$$
\begin{aligned}
& \Psi_{\nu_{ \pm}}= \operatorname{Exp}\left[\left(\frac{\mu_{\ell}}{2 \hbar}\left(48-\frac{1}{3 \mathrm{c}_{\ell}}\right)\left(\xi_{1}+\xi_{2}\right)+\frac{\xi_{3}}{\hbar}+\frac{\mathrm{a}_{4} \mathrm{u}_{1}+\mathrm{a}_{5} \mathrm{u}_{2}+\mathrm{a}_{6} \mathrm{u}_{3}}{\hbar}\right) \mathrm{a}_{3}\right] \times \\
& \mathrm{K}_{\nu_{-}}\left(\frac{4}{\hbar} \sqrt{6 \mathrm{~V}_{1} \mu_{\ell}} \mathrm{e}^{-\frac{\xi_{1}}{2}}\right) \mathrm{K}_{\nu_{+}}\left(\frac{4}{\hbar} \sqrt{6 \mathrm{~V}_{2} \mu_{\ell}} \mathrm{e}^{-\frac{\xi_{2}}{2}}\right) .
\end{aligned}
$$

\section{Quantum Anisotropic Quintessence Case}

Lastly, we are going to consider the quantum version of the anisotropic quintessence like case. As in the previous two subsections, what we want is to obtain an equation of the form $\mathcal{H} \Psi\left(\xi_{\mathrm{i}}\right)=0$, to achieve this we introduce the standard prescription $\Pi_{q}^{\mu}=-i \hbar \partial_{q^{\mu}}$ in $(99)$, obtaining

$$
\begin{aligned}
& {\left[-\frac{\hbar^{2}}{\nu_{\ell}} \frac{\partial^{2}}{\partial \xi_{1}^{2}}-\frac{\hbar^{2}}{\nu_{\ell}} \frac{\partial^{2}}{\partial \xi_{2}^{2}}-\hbar^{2}\left(24+\frac{1}{3 \nu_{\ell}}\right)\left(\frac{\partial^{2}}{\partial \xi_{3} \partial \xi_{1}}+\frac{\partial^{2}}{\partial \xi_{3} \partial \xi_{2}}\right)-\hbar^{2}\left(12+\frac{1}{18 \nu_{\ell}}\right) \frac{\partial^{2}}{\partial \xi_{3}^{2}}\right.} \\
& \left.+6 \hbar^{2}\left(\frac{\partial^{2} \Psi}{\partial \mathrm{u}_{1}^{2}}+\frac{\partial^{2} \Psi}{\partial \mathrm{u}_{2}^{2}}+\frac{\partial^{2} \Psi}{\partial \mathrm{u}_{3}^{2}}\right)-24 \mathrm{~V}_{1} \mathrm{e}^{-\xi_{1}}-24 \mathrm{~V}_{2} \mathrm{e}^{-\xi_{2}}\right] \Psi=0,
\end{aligned}
$$

we can see that the scalar potential does not depend on the coordinates $\xi_{3}, \mathrm{u}_{\mathrm{i}}$, consequently we propose the following ansatz for the wave function $\Psi\left(\xi_{1}, \xi_{2}, \xi_{3}\right)=\mathrm{e}^{\left(\mathrm{b}_{3} \xi_{3}+\mathrm{b}_{4} \mathrm{u}_{1}+\mathrm{b}_{5} \mathrm{u}_{2}+\mathrm{b}_{6} \mathrm{u}_{3}\right) / \hbar} \mathcal{A}\left(\xi_{1}\right) \mathcal{B}\left(\xi_{2}\right)$ where $\mathrm{b}_{\mathrm{i}}($ with $\mathrm{i}=3,4,5,6)$ are an arbitrary constant. Applying and dividing by the ansatz in 141 we get

$$
\begin{aligned}
& -\frac{\hbar^{2}}{\nu_{\ell} \mathcal{A}} \frac{\mathrm{d}^{2} \mathcal{A}}{\mathrm{d} \xi_{1}^{2}}-\frac{\hbar^{2}}{\nu_{\ell} \mathcal{B}} \frac{\mathrm{d}^{2} \mathcal{B}}{\mathrm{d} \xi_{2}^{2}}-\mathrm{b}_{3} \hbar\left(48-\frac{1}{3 \mathrm{c}_{\ell}}\right)\left(\frac{1}{\mathcal{A}} \frac{\mathrm{d} \mathcal{A}}{\mathrm{d} \xi_{1}}+\frac{1}{\mathcal{B}} \frac{\mathrm{d} \mathcal{B}}{\mathrm{d} \xi_{2}}\right)-\mathrm{b}_{3}^{2}\left(16-\frac{1}{18 \mathrm{c}_{\ell}}\right) \\
& +6 b_{0}^{2}-24 V_{1} e^{-\xi_{1}}-24 V_{2} e^{-\xi_{2}}=0,
\end{aligned}
$$

with $b_{0}^{2}=b_{4}^{2}+b_{5}^{2}+b_{6}^{2}$, separating the equations we have that

$$
\begin{aligned}
\frac{\mathrm{d}^{2} \mathcal{A}}{\mathrm{d} \xi_{1}^{2}} & +\frac{b_{3} \nu_{\ell}}{\hbar}\left(48-\frac{1}{3 c_{\ell}}\right) \frac{d \mathcal{A}}{d \xi_{1}} \\
& +\frac{\nu_{\ell}}{\hbar^{2}}\left(b_{3}^{2}\left(8-\frac{1}{36 c_{\ell}}\right)-3 b_{0}^{2}-\alpha^{2}+24 V_{1} e^{-\xi_{1}}\right) \mathcal{A}=0 \\
\frac{\mathrm{d}^{2} \mathcal{B}}{\mathrm{d} \xi_{2}^{2}} & +\frac{b_{3} \nu_{\ell}}{\hbar}\left(48-\frac{1}{3 c_{\ell}}\right) \frac{d \mathcal{B}}{d \xi_{2}} \\
& +\frac{\mu_{\ell}}{\hbar^{2}}\left(b_{3}^{2}\left(8-\frac{1}{36 c_{\ell}}\right)-3 b_{0}^{2}+\alpha^{2}+24 V_{2} e^{-\xi_{2}}\right) \mathcal{B}=0
\end{aligned}
$$


where $\alpha^{2}$ is the separation constant. These last two equations are similar to those of the quantum quintom like case 132 and 133 . Proceeding in a similar fashion as the previous subsection (IVB), we make the following identifications

$$
\begin{aligned}
\lambda & =-1, \\
\mathrm{a} & =\frac{b_{3} \nu_{\ell}}{\hbar}\left(48-\frac{1}{3 c_{\ell}}\right), \\
\mathrm{b}_{1,2} & =\frac{\nu_{\ell}}{\hbar^{2}} 24 V_{1,2}, \\
\mathrm{c}_{\mp} & =\frac{\nu_{\ell}}{\hbar^{2}}\left(b_{3}^{2}\left(8-\frac{1}{36 c_{\ell}}\right)-3 a_{0}^{2} \mp \alpha^{2}\right),
\end{aligned}
$$

and conclude that the solutions are given by the ordinary Bessel function $J_{\nu}$ with order $\nu_{\mp}=\sqrt{\left(\mathrm{a}^{2} / \nu_{\ell}\right)+4 \mathrm{c}_{\mp}}$. Thus, the wave function becomes

$$
\begin{aligned}
\Psi_{\nu_{ \pm}}= & \operatorname{Exp}\left[\left(\frac{\nu_{\ell}}{2 \hbar}\left(48-\frac{1}{3 \mathrm{c}_{\ell}}\right)\left(-\xi_{1}-\xi_{2}\right)+\frac{\xi_{3}}{\hbar}\right) \mathrm{b}_{3}+\frac{\mathrm{b}_{4} \mathrm{u}_{1}+\mathrm{b}_{5} \mathrm{u}_{2}+\mathrm{b}_{6} \mathrm{u}_{3}}{\hbar}\right] \times \\
& \mathrm{J}_{\nu_{-}}\left(\frac{4}{\hbar} \sqrt{6 \mathrm{~V}_{1} \nu_{\ell}} \mathrm{e}^{-\frac{\xi_{1}}{2}}\right) \mathrm{J}_{\nu_{+}}\left(\frac{4}{\hbar} \sqrt{6 \mathrm{~V}_{2} \nu_{\ell}} \mathrm{e}^{-\frac{\xi_{2}}{2}}\right) .
\end{aligned}
$$

\section{FINAL REMARKS}

In this work we have studied the anisotropic Bianchi type model in the chiral cosmology setup in a twofold way. In the first cosmological model we consider two scalar fields but a single term of the potential. In the second one, additionally to the two scalar fields, we also consider both terms in the potential. For both models we did a classical and quantum treatment, obtaining exact analytical solutions for both scenarios.

In the first model, which can be thought as a quintessence plus k-essence model, our findings show that the volume of the universe grows in an accelerated manner for each of the three exact solutions that were found. This feature can be seen from Fig. (1), where solutions for $\lambda_{1}<\sqrt{3}$ and $\lambda_{1}=\sqrt{3}$ have a similar behavior whereas the solution for $\lambda>\sqrt{3}$ has a more faster evolution. After a certain amount of time, the three solutions stabilized at the same value of -1 . Also, because of the ratio of the shear to scalar expansion bound: $\sigma / \theta \leq 0.3$ [82, we were able to constrain the value of the anisotropic parameter $\overline{\mathrm{A}}_{\mathrm{m}}$ for two of the solutions, however the anisotropy continue, because for the cases $\lambda_{1}>\sqrt{3}$ and $\lambda_{1}=\sqrt{3}$ the anisotropic parameter becomes $\overline{\mathrm{A}}_{\mathrm{m}} \leq 3.54$. In the quantum regime we were also able to find exact solutions. For the particular case of $\lambda_{1}<\sqrt{3}$ we found that the wave function has a damped behavior as the scale factor evolves, as can be seen in Fig. (2), this distinctive mark have also been reported in [68, 80, 88, In contrast with the isotropic treatment [80, we found that the anisotropies shrink the probability density of the wave function. For the solution $\lambda_{1}>\sqrt{3}$, it is found that the damped behavior still exists, but the parameter $\mathrm{Q}$ acts as a retarder (for negative values) for the wave function and the length over the axis were the field evolves is compressed as shown in Fig.(3), signaling that the inflation epoch should also be retarded in time. In this case the anisotropies shrink the probability density along the evolution of the scalar field. Finally, equation (129) depicts the quantum solution for the case $\lambda_{1}=\sqrt{3}$.

For the second model under study, we consider both potential terms, in addition to the two scalar fields. In this setup two possible avenues were distinguished: a quintom one and a quintessence one. Classical exact solutions for the former are given by equations (97), while the solutions for the latter are given by equations (110). For the quantum counterpart exact solutions were also obtained. The quantum solutions for both the anisotropic quintom case and 
the anisotropic quintessence case, are given in terms of exponential functions (that has the anisotropic information) multiplied by the modified Bessel function $K_{ \pm \nu}$ and the ordinary Bessel function $J_{ \pm \nu}$, as depicted in Eq. 140 and Eq. 150, respectively.

\section{Acknowledgments}

This work was partially supported by PROMEP grants UGTO-CA-3. J.S. is partially supported SNI-CONACYT. This work is part of the collaboration within the Instituto Avanzado de Cosmología and Red PROMEP: Gravitation and Mathematical Physics under project Quantum aspects of gravity in cosmological models, phenomenology and geometry of space-time. Many calculations where done by Symbolic Program REDUCE 3.8.

[1] D. J. Schwarz, C. J. Copi, D. Huterer and G. D. Starkman, Class. Quant. Grav. 33, (2016), no.18, 184001.

[2] V. T. Gurovich and A. A. Starobinsky, Sov. Phys. JETP 50 (1979), 844-852.

[3] D. Müller, A. Ricciardone, A. A. Starobinsky and A. Toporensky, Eur. Phys. J. C 78 (2018) no.4, 311.

[4] A. Y. Kamenshchik, E. O. Pozdeeva, A. A. Starobinsky, A. Tronconi, G. Venturi and S. Y. Vernov, Phys. Rev. D 97 (2018) no.2, 023536.

[5] M. C. Pookkillath, A. De Felice and A. A. Starobinsky, JCAP 07 (2020), 041.

[6] A. A. Starobinsky, S. V. Sushkov and M. S. Volkov, Phys. Rev. D 101 (2020) no.6, 064039.

[7] T.S. Pereira, C. Pitrou and J.P. Uzan, JCAP 09, (2007) 006.

[8] C. Pitrou, T.S. Pereira and J.P. Uzan, JCAP 04, (2008) 004.

[9] T. Pereira and C. Pitrou, Comptes Rendus Physique 16, (2015) 1027-1037.

[10] A.E. Gumrukcuoglu, C.R. Contaldi and M. Peloso, JCAP 11, (2007) 005.

[11] A. A. Starobinsky, Phys. Lett. B 91 (1980), 99-102.

[12] A.D. Linde, Phys. Lett. B 108, (1982) 389-193.

[13] A.D. Linde, Phys. Lett. B 129, (1983) 177-181.

[14] J.D. Barrow, Phys. Rev. D 48, (1983) 1585-1590.

[15] J.D. Barrow and P. Saich, Class. Quant. Grav. 10, (1993) 279-283.

[16] P.J.E. Peebles and B. Ratra, Astrophys. J. Lett. 325, (1988) L17 .

[17] S. Tsujikawa, Class. Quant. Grav. 30, (2013) 214003.

[18] A.R. Liddle and R.J. Scherrer, Phys. Rev. D 59, (1999) 023509.

[19] V. Sahni and L.M. Wang, Phys. Rev. D 62, (2000) 103517.

[20] T. Matos and L.A. Ureña-López, Phys. Rev. D 63, (2001) 063506.

[21] L. A. Ureña-López, J. Phys. Conf. Ser. 761 (2016) no.1, 012076

[22] P.J.E. Peebles and A. Vilenkin, Phys. Rev. D 59, (1999) 063505.

[23] J. Haro, J. Amorós and S. Pan S, Phys. Rev. D 93, (8) (2016) 084018.

[24] J. Haro, J. Amorós and S. Pan, Phys. Rev. D 94, (6) (2016) 064060.

[25] E. Elizalde, S. Nojiri and S.D. Odintsov, Phys. Rev. D 70, (2004) 043539.

[26] A. A. Coley and R. J. van den Hoogen, Phys. Rev. D 62, (2000) 023517.

[27] E. J. Copeland, A. Mazumdar and N. J. Nunes, Phys. Rev. D 60, (1999) 083506.

[28] G. Calcagni and A.R. Liddle, Phys. Rev. D 77, (2008) 023522.

[29] S. Yokoyama, T. Suyama and T. Tanaka, Phys. Rev. D 77, (2008) 083511.

[30] T. Chiba and M. Yamaguchi, JCAP 0901, (2009) 019.

[31] A.R. Liddle, A. Mazumdar and F.E. Schunck, Phys. Rev. D 58, (1998) 061301.

[32] Y.F. Cai, E.N. Saridakis, M.R. Setare and J.Q. Xia, Phys. Rept. 493, (2010) 1-60.

[33] M.R. Setare and E.N. Saridakis, Int. J. Mod. Phys. D 18, (2009) 549-557.

[34] R. Lazkoz, G. Leon and I. Quiros, Phys. Lett. B 649, (2007) 103-110.

[35] G. Leon, A. Paliathanasis and J.L. Morales-Martínez, Eur. Phys. J. C 78, (9) (2018) 753.

[36] L.P. Chimento, M. Forte, R. Lazkoz and M.G. Richarte, Phys. Rev. D 79, (2009) 043502.

[37] A.D. Lindle, Phys. Rev. D 49, (1994) 784.

[38] E.J. Copeland, A.R. Liddle, D.H. Lyth, E.W. Steward and D. Wands, Phys. Rev. D 49, (1994) 6410.

[39] S.A. Kim and A.R. Liddle, Phys. Rev. D 74, (2006) 023513.

[40] J. Socorro and O.E. Núñez, Eur. Phys. Journal Plus 132, (2017) 168.

[41] D. Wands, Lecture Notes in Physics 738 (Springer, Berlin, Heidelberg, 2008).

[42] J.R. Bond, L. Kofman, S. Prokushkin and P.M. Vaudrevange, Phys. Rev. D 75, (2007) 123511.

[43] K. Inomata, M. Kawasaki, K. Mukaida and T.T. Yanagida, Phys. Rev. D 97, (4) (2018) 043514.

[44] O.E. Núñez, J. Socorro and R. Hernández-Jiménez, Astrophys. Space Sci. 364, (2019) 69. 
[45] J.D. Barrow, Phys. Rev. D 51, (1995) 2729.

[46] P.G. Ferreira and M. Joyce, Phys. Rev. D 58, (1998) 023503.

[47] E.J. Copeland, M. Sami and S. Tsujikawa, Int. J. Mod. Phys. D 15, (2006) 1753.

[48] E.J. Copeland, A.R. Liddle and D. Wands, Phys. Rev. D 57, (1998) 4686.

[49] E.J. Copeland, T. Barreiro and N. Nunes, Phys. Rev. D 61, (2000) 127301.

[50] D. Saez-Gomez, arXiv:0812.1980 [hep-th]].

[51] M. Capone, C. Rubano and P. Scudellaro, Eur. Phys. Lett. 73, (2006) 149-155.

[52] E.W. Kolb and M.S. Turner, The Early Universe (Addison-Wesley publishing Co., Illinois, 1998).

[53] R. Myrzakulov, L. Sebastiani and S. Vagnozzi, Eur. J. Phys. Plus 75, (2015) 444.

[54] S.V. Chervon, Russ. Phys. J. 38, (1995) 539-543.

[55] S.V. Chervon, Quantum Matter 2, (2) (2013) 71-82.

[56] D.I. Kaiser and E.I. Sfakianakis, Phys. Rev. Lett. 112, (2014) 011302.

[57] I.V. Fomin, J. Phys. Conf. Ser. 918, (1) (2017) 012009.

[58] S.V. Chervon, I.V. Fomin, E.O. Pozdeeva, M. Sami and S.Y. Vernov, Phys. Rev. D 100, (2019) 063522.

[59] A. Paliathanasis, G. Leon and S. Pan, Gen. Rel. and Grav. 51, (2019) 106.

[60] A. Paliathanasis, Class. and Quant. Grav. 37, (19) (2020) 195014.

[61] A. Paliathanasis and G. Leon, Eur. Phys. J. C 80, (2020) 847.

[62] K. Bamba, S. Capozziello, S. Nojiri and S.D. Odintsov, Astrophys. Space Sci. 342, (2012) 155-228.

[63] N. Dimakis and A. Paliathanasis, arXiv:2001.09687 [gr-qc].

[64] N. Dimakis, A. Paliathanasis, P.A. Terzis and T. Christodoulakis, Eur. Phys. J. C 79, (7) (2019) 618.

[65] A. Paliathanasis and M. Tsamparlis, Phys. Rev. D 90, (4) (2014) 043529.

[66] V. Folomeev, Int. J. Mod. Phys. D 16, (2007) 1845-1852.

[67] J. Socorro, M. Sabido, W. Ramírez, Máximo G. Agüero, Inflación cosmológica vista desde la mecánica cuántica supersimétrica, in: Máximo A. Agüero (Ed.), Procesos no lineales en la ciencia y la sociedad, (Notabilis Scientia, Granados, 2013), pages. 99-121, Chapter in Spanish book.

[68] J. Socorro, O.E. Núñez and R. Hernández-Jiménez, Phys. Lett. B 809, (2020) 135667.

[69] A.A. Andrianov, O. O. Novikov and C. Lan, Teor. Mat. Fiz. 184, (3) (2015) 380-391.

[70] A.M. Galiakhmetov, Int. J. Mod. Phys. D 23, (2014) 1450034.

[71] R.R. Abbyazov and S.V. Chervon, Mod. Phys. Lett. A 28, (2013) 1350024.

[72] S.V. Chervon, R. R. Abbyazov and S.V. Kryukov, Russ. Phys. J. 58,(5) (2015) 597-605.

[73] F. Cicciarella, J. Mabillard, M. Pieroni and A. Ricciardone,JCAP 09, (2019) 044.

[74] Y. Herfray, K. Krasnov and Y. Shtanov, Class. Quant. Grav. 33, (2016) 235001.

[75] G. Leon and F.O.F. Silva,Class. Quant. Grav. 37, (24) (2020) 245005.

[76] D.I. Kaiser, Phys. Rev. D 81, (2010) 084044.

[77] D.I. Kaiser and E.I. Sfakianakis, Phys. Rev. Lett. 112, (1) (2014) 011302.

[78] A. Beesham, S.V. Chervon, S.D. Maharaj and A.S. Kubasov, Quant. Matt. 2, (2013) 388-395.

[79] J. Socorro, S. Pérez-Payán, A. Espinoza-García and L.R. Díaz-Barrón, Astrophys. Space Sci. 365, (6) (2020) 93.

[80] J. Socorro, S. Pérez-Payán, R. Hernández, A. Espinoza-García and L. R. Díaz-Barrón, arXiv:2012.11108 [gr-qc]]. Accepted for publication in CQG. https://doi.org/10.1088/1361-6382/abfed7.

[81] S.K. Tripathy, Astrophys. Sp. Sci. 340, (2012) 211.

[82] A. Pradhan, H. Amirhashchi and B. Saha, Int. J. Theor. Phys. 50, (2011) 2923-2938.

[83] G.W. Gibbons and L. P. Grishchuk, Nucl. Phys. B 313, (1989) 736.

[84] Li Zhi Fang and Remo Ruffini, Editors, Quantum Cosmology, Advances Series in Astrophysics and Cosmology Vol. 3 (World Scientific, Singapore, 1987).

[85] J. Hartle, and S.W. Hawking, Phys. Rev. D 28, (1983) 2960.

[86] S.W. Hawking, Nucl. Phys. B 239, (1984) 257.

[87] V.F. Zaitsev and A.F. Polyanin, Handbook of Exact Solutions for Ordinary Differential Equations (Taylor and Francis Editorial, 2002).

[88] J. Socorro, O.E. Núñez and R. Hernández-Jiménez, Adv. Math. Phys. 20183468381. 